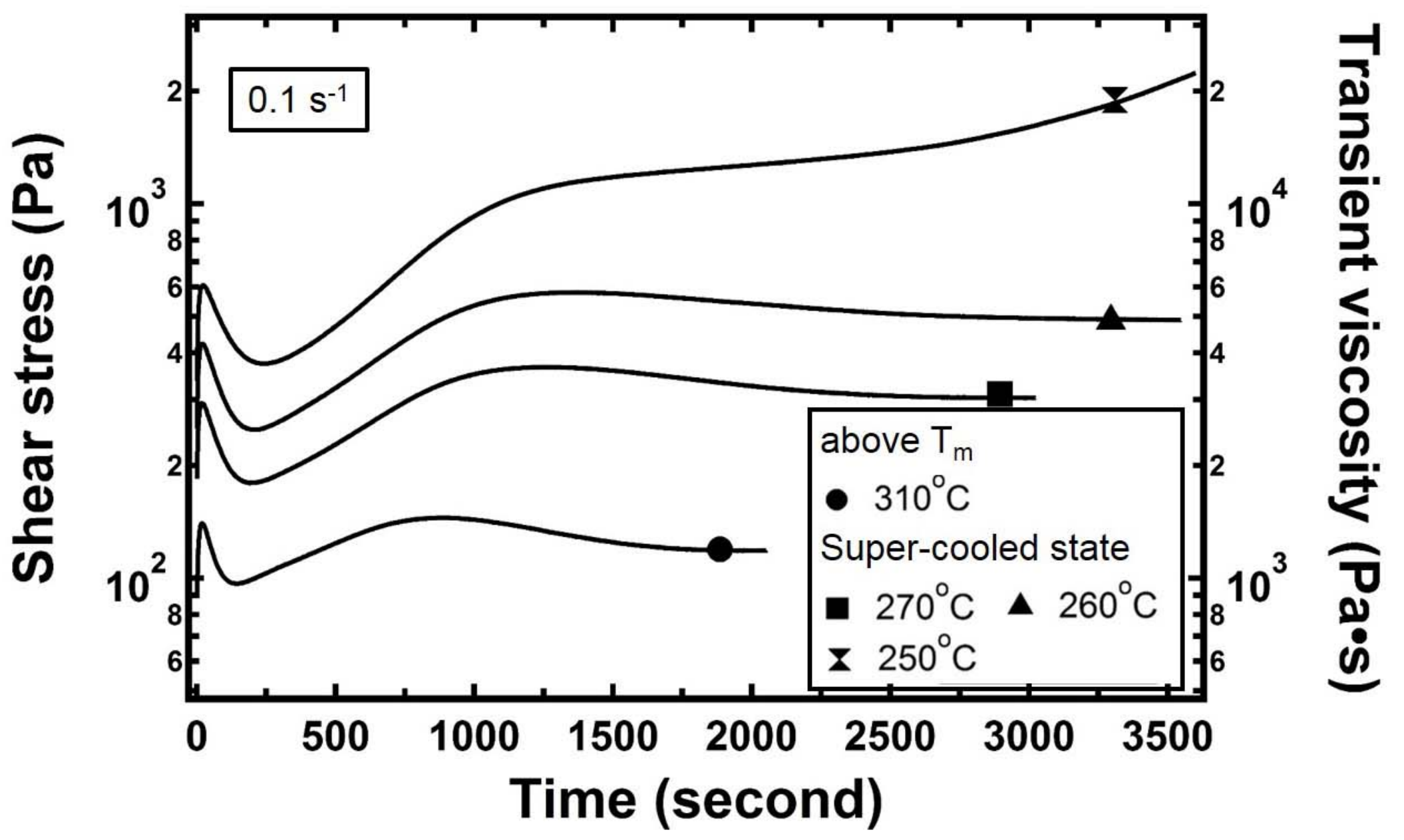




\title{
The Transient Shear Rheology of a Thermotropic Liquid Crystalline Polymer in the Super- cooled State
}

\author{
Chen Qian ${ }^{\mathrm{a}}$, Donald G. Baird ${ }^{\mathrm{a}^{*}}$ \\ aDepartment of Chemical Engineering, Virginia Polytechnic Institute and State University, \\ Blacksburg, VA 24061
}

\section{Abstract}

To determine the processing temperature of a representative thermotropic liquid crystalline polymer (TLCP) in the super-cooled state, small amplitude oscillatory shear (SAOS), the startup of shear flow and differential scanning calorimetry (DSC) measurements were performed. Good agreement on the onset crystallization temperature was obtained from the SAOS and the startup of shear flow. Compared with DSC, the rheological measurements were more sensitive for detecting crystallization in the super-cooled state. The rheological study suggested that crystallization did not occur unless the TLCP was cooled $30^{\circ} \mathrm{C}$ below its melting point, and its viscosity was significantly increased as the material was super cooled. Additionally, super cooling the TLCP did not significantly affect the relaxation of shear stress after preshearing. However, the recovery of transient shear stress in the interrupted shear measurements was suppressed to a great extent in the super-cooled state.

Key words: Thermotropic liquid crystalline polymer, Transient rheology, Super-cooled state 


\section{Introduction}

Thermotropic liquid crystalline polymers (TLCPs) are high performance engineering thermoplastics, which offer a balance of properties unmatched by most other resins. TLCPs are well known for their outstanding mechanical properties. For example, the specific tensile modulus of a commercial TLCP fiber, Vectran ${ }^{\circledR}$, is 5.6 times higher than steel, and its specific strength is 1.8 times higher [1]. In addition to their mechanical properties, the highly compact structure of liquid crystalline polymers significantly decreases the solubility of gas molecules, making TLCPs one of the least permeable thermoplastics [2-3]. Weinkauf and Paul [4-6] systematically investigated the transport phenomena of various gases in the a TLCP prepared from $73 \mathrm{~mol} \%$ hydroxybenzoic acid (HBA) and 27 mol\% hydroxynaphthoic acid (HNA) (commercial name Vectra A950), and they showed the permeability of $\mathrm{O}_{2}$ in the $73 / 27 \mathrm{HBA} / \mathrm{HNA}$ was more than two orders of magnitude lower relative to the commonly used barrier resin poly(ethylene terephthalate) (PET). The exceptional barrier properties make TLCPs promising candidates for some packaging applications [7-8] and for hydrogen and oxygen storage.

Commercial TLCPs exhibit inherently low viscosity relative to many other conventional thermoplastics. The low melt viscosity gives rise to enhanced processability of TLCPs using techniques such as injection molding. However, it is detrimental to processes requiring high melt strength, such as extrusion blow molding, film blowing, thermoforming and multilayer coextrusion [8].

Several methods have been developed to modify the rheological characteristics of TLCPs, such that their viscosity can be enhanced. Carbon fillers, including carbon black, graphite and carbon fiber, have been added to TLCP matrices to effectively increase the viscosity [9-11]. 
Nevertheless, it is uncertain whether the addition of fillers will influence the great barrier properties of TLCPs, as voids may exist between the fillers and matrix due to the lack of adhesion between the two phases.

Efforts were also made on tuning the rheological properties of TLCPs by chemical modification. Li et al. [12] conducted melt-state reaction using a HBA/HNA TLCP and triphenyl phosphite. In this case one triphenyl phosphite molecule could react with up to three TLCP chainend functionalities. It was found the shear viscosity can be enhanced by a factor of 20 over the neat resin. In a later publication, the authors reported the reaction between TLCP and diepoxy reagent in the molten state. The product was used to prepare high quality multilayer films [8]. Corallo et al. [13-14] patented the extrusion blow molding from both neat and glass fiber filled 73/27 HBA/HNA. In this case the viscosity enhancement resulted from the heat treatment of the raw materials. When TLCP molecules were exposed to air, polymerization occurred at elevated temperatures in the solid state, giving rise to molecules with higher molecular weight and viscosity. However, these two patents did not specify whether solid state polymerization led to linear or cross linked polymers.

Done and Baird [15-16] developed a unique method to increase the viscosity of TLCPs by first raising the temperature above the melting point $\left(\mathrm{T}_{\mathrm{m}}\right)$ to exclude all solid crystalline structure and then lowering the temperature below $\mathrm{T}_{\mathrm{m}}$ to super cool the materials [17]. The processing temperature window in the super-cooled state was determined by small amplitude oscillatory shear measurements in the temperature ramp mode. When being processed in the super-cooled state, the viscosity and melt strength of TLCPs were effectively improved. This resulted in the successful extrusion blow molding and film blowing of TLCPs. 
The shear rheology of commercial TLCPs has been well documented under conventional processing conditions above $\mathrm{T}_{\mathrm{m}}$ [18-29]. Other than the 73/27 HBA/HNA, two other commercial TLCPs have been widely studied. One is a copolyester synthesized from 40 mol\% PET and 60 mol\% HBA. The other is a polyesteramide containing $60 \mathrm{~mol} \% \mathrm{HNA}, 20 \mathrm{~mol} \%$ terephthalic acid (TA) and 20 mol\% 4-aminophenol (commercial name Vectra B950). TLCPs have demonstrated some unusual flow behavior, such as the three region flow curve $[19,21,23,26]$, the debatable negative primary normal stress difference [24-26, 28, 30], the scaling of shear stress growth curves with strain at different shear rates [18-19, 23], and the strong dependence of transient rheological behaviors on flow history [18, 27].

The complicated rheology of TLCP melts originates from their structured fluid nature. TLCP molecules form the nematic phase with a polydomain structure in the molten state [20-21, 31]. Within each domain, the orientations of the rigid, rod-like molecules are relatively uniform. However, the domain orientations are distributed heterogeneously in space. Upon shearing, the structure evolution of TLCP melts involve both the reorientation of molecules and the rearrangement of the domain structure [18, 20-21, 27, 29]. Viola and Baird [27] compared the simulation data with the experimental results for 40/60 PET/HBA. They concluded that instead of the shear induced orientation of the molecules, the domain structure change was responsible for the stress growth response in the startup of shear flow. On the other hand, Beekmans et al. [18] studied the influence of mechanical history on the stress growth of 60/20/20 HNA/TA/ aminophenol. The startup and relaxation stress transients were at least partially attributed to the rotation and orientation of the molecules. Therefore, the origin for the complex transient rheological behavior could be very complicated and highly material dependent. 
McLeod and Baird [32] melted the TLCP containing HBA/TA/HQ/HQ-derivatives (HX8000) first, and cooled the material down at various cooling rates to track the crystallization temperatures, using both small amplitude oscillatory shear measurements and DSC analysis. However, the crystallization temperature alone is not enough to guide processing in the supercooled state, it is of equal importance to know how fast the TLCPs crystallize at temperatures below the melting points. Done and Baird [17] studied the crystallization behaviors of three different TLCPs (40/60 PET/HBA, 20/80 PET/HBA and 73/27 HBA/HNA). Moreover, the onset crystallization temperatures of these TLCPs were investigated in the super-cooled state, which were obtained from both dynamic mechanical measurements and DSC. Nevertheless, the flow behavior of the TLCPs under steady shear was not reported. The first objective of our study is to determine the onset crystallization temperatures in the super-cooled, using the copolyester of HBA/TA/HQ/HQ-derivatives (HX8000) as a representative material. The practical significance of this part of the work is to provide guidance for processing. To achieve this objective, we used not only small amplitude oscillatory shear and DSC measurements, but transient experiments at the startup of shear flow. As far as we know, this is the first time the transient stress growth of a TLCP is measured in the super-cooled state. We also aim to quantify the viscosity increment as a TLCP is cooled to the super-cooled state. The viscosity at low shear rates indicates melt strength, which is critical to melt processing.

Additionally, the transient rheology of TLCPs above $\mathrm{T}_{\mathrm{m}}$ has been well documented, which indicates the structure evolution of TLCPs. However, the transient rheology of TLCPs in the supercooled state has yet to be investigated. Our second objective is to determine how cooling the TLCP to the super-cooled state will affect the transient rheological behavior. 


\section{Experimental}

\subsection{Materials}

Zenite HX8000 was provided by DuPont and was used as received. The Zenite series of TLCPs is believed to be synthesized from different ratios of terephthalic acid (TA), 4hydroxybenzoic acid (HBA), hydroquinone (HQ) and hydroquinone derivatives (HQ-derivatives) [32]. Nevertheless, the specific monomer ratio for HX8000 is unknown. The HX8000 has the clearing temperature above its melting point, and the polymer degrades before the nameticisotropic transition occurs.

\subsection{Differential Scanning Calorimetry}

DSC measurement in the temperature ramp mode was carried out using a TA instrument's Discovery DSC. The temperature ramp test was used to obtain the melting point, as well as the crystallization temperatures of the TLCP. Ca. $10 \mathrm{mg}$ of sample was equilibrated at $350^{\circ} \mathrm{C}$ for 1 min, the material was cooled to $40^{\circ} \mathrm{C}$ (first cooling scan), and then heated back up to $350^{\circ} \mathrm{C}$ ( $\operatorname{second}$ heating scan). A constant rate of $20^{\circ} \mathrm{C} / \mathrm{min}$ was applied in both heating and cooling scans, and the measurement was performed under a dry nitrogen environment. It was found the melting and crystallization temperatures were independent of the equilibration time at $350^{\circ} \mathrm{C}$. To avoid the chemical change of the material at high temperature, such as degradation and transesterification, the equilibration time was set as $1 \mathrm{~min}$ [32-34]. The melting point as well as the melting enthalpy were calibrated using indium.

DSC tests were also performed in the isothermal time sweep mode. Ca. $10 \mathrm{mg}$ of HX8000 was first equilibrated at $310^{\circ} \mathrm{C}$ for 10 mins and then cooled to 270,260 and $250^{\circ} \mathrm{C}$ at $20^{\circ} \mathrm{C} / \mathrm{min}$. Heat flow was traced as the TLCP was held at a given temperature. 


\subsection{Rheological Measurements}

Small amplitude oscillatory shear measurements were performed in the temperature ramp mode, using a TA instrument's ARES-G2 rheometer with $25 \mathrm{~mm}$ diameter parallel disks fixtures. HX8000 pellets were dried in a vacuum oven at $100^{\circ} \mathrm{C}$ for at least $24 \mathrm{hr}$ prior to testing. In the sample loading process, pellets were directly loaded between the parallel disks and melted for 3 $\mathrm{min}$, then the gap was closed to $0.2 \mathrm{~mm}$ at a rate of $2 \mu \mathrm{m} / \mathrm{s}$. With $0.2 \mathrm{~mm}$ gap, each sample was brought to the starting temperature of $310^{\circ} \mathrm{C}$ and equilibrated for $10 \mathrm{~min}$. After equilibration the temperature was lowered at a rate of $20^{\circ} \mathrm{C} / \mathrm{min}$. Oscillatory shear with $2.5 \%$ strain and $10 \mathrm{rad} / \mathrm{s}$ angular frequency were applied, and the storage modulus G' and loss modulus G” were monitored as a function of temperature. All measurements were performed within a dry nitrogen environment.

Small amplitude oscillatory shear tests were also conducted in the time sweep mode. The same sample loading procedures adopted were the same as those described in the temperature ramp mode. TLCP pellets were first melted at $310^{\circ} \mathrm{C}$, and then the temperature was lowered to 270,260 and $250^{\circ} \mathrm{C}$ at $20^{\circ} \mathrm{C} / \mathrm{min}$. The samples were then sheared at a frequency of $10 \mathrm{rad} / \mathrm{s}$ with $2.5 \%$ strain. The growths of G' and G" were observed as a function of time.

For the transient rheological measurements, parallel disk fixtures with a $0.2 \mathrm{~mm}$ gap were used. The sample loading procedures were essentially the same as those used in the small amplitude oscillatory shear tests described above. Besides $310^{\circ} \mathrm{C}$, which was above $\mathrm{T}_{\mathrm{m}}$, measurements were also carried out at temperatures in the super-cooled state, namely 270,260 and $250^{\circ} \mathrm{C}$. For experiments in the super-cooled state, samples were first equilibrated at $310^{\circ} \mathrm{C}$, and then they were cooled below the melting point of $280^{\circ} \mathrm{C}$ at $20^{\circ} \mathrm{C} / \mathrm{min}$. Once the new temperature is reached, the material is allowed to equilibrate for another 3 mins to ensure temperature equilibration is achieved. 
To evaluate the time consumed for the center part of the sample to be cooled to the new temperatures, we assume the TLCP has similar thermal conductivity and heat capacity relative to PET, and the temperature of the sample remains constant during cooling. Following the method of Baird and Collias [35], it is shown the TLCP will be cooled to the new temperature within 30s. Therefore, we are confident the entire sample is re-equilibrated in 3 mins.

To compensate for the gap increase during cooling, the "move stage to maintain starting gap" function of the ARES-G2 rheometer was activated. During the cooling, the upper fixture moved down automatically. The distance the upper fixture traveled was based on the thermal expansion coefficient of the fixtures as well as the temperature decreased. Therefore, the gap was maintained at $0.2 \mathrm{~mm}$ when the measurements started.

Five different types of transient experimental protocols were applied. The first type of transient measurements conducted was the startup of shear flow. A shear rate, $\dot{\gamma}$, of either 0.1 or $0.5 \mathrm{~s}^{-1}$ was applied until the TLCP reached a steady state. The second type of test was the relaxation after flow cessation. The material was first presheared at $0.5 \mathrm{~s}^{-1}$ for $1600 \mathrm{~s}$ to a steady state, then shearing was stopped and the shear stress was allowed to relax for $1000 \mathrm{~s}$. In the third set of measurements, after preshearing at $0.5 \mathrm{~s}^{-1}$ for $1600 \mathrm{~s}$, oscillatory shear of $2.5 \%$ strain and $10 \mathrm{rad} / \mathrm{s}$ frequency was immediately applied. The fourth type of experiment was that of the interrupted shear. After preshearing at $0.5 \mathrm{~s}^{-1}$ for $1600 \mathrm{~s}$, TLCP relaxed for various periods of 50, 200 and 500 $\mathrm{s}$, then shearing was resumed at $0.5 \mathrm{~s}^{-1}$. In the last type of measurement, flow reversal was applied. That is, after the same preshearing protocol was applied as in the interrupted shear, the shearing direction was reversed after $0,50,200$ and $500 \mathrm{~s}$ of relaxation $\left(\dot{\gamma}=0.5 \mathrm{~s}^{-1}\right)$. To better illustrate the methods and the corresponding transient stress response, a schematic representation is provided in 
Fig 1. The stress response in Fig 1 is not obtained from any real measurement, and it does not refer to any particular testing condition.

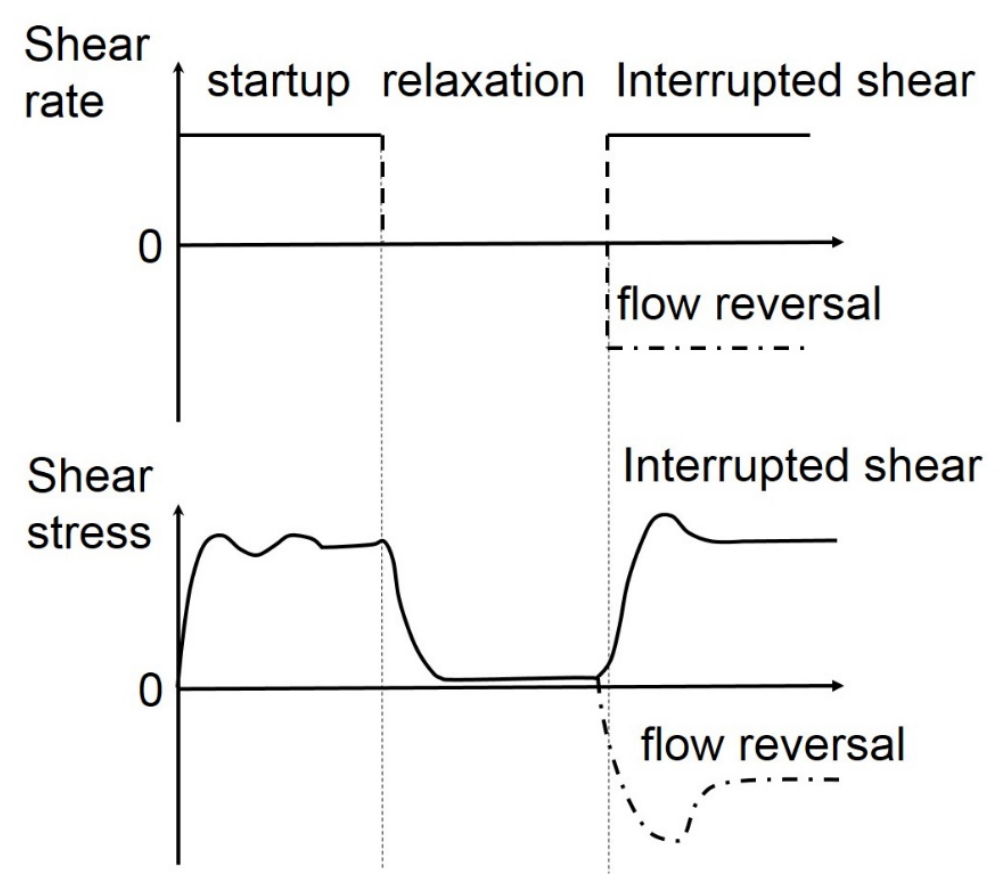

Figure 1: A schematic representation of the transient shearing protocol and the corresponding transient shear stress response. The stress response is not obtained from any real measurement, and it does not refer to any particular conditions.

\section{Results and Discussion}

\subsection{DSC measurements}

The results of DSC heating and cooling scans are shown in Fig 2. The heating scan indicates the high temperature end of the melting endotherm is $276^{\circ} \mathrm{C}$, which is defined as the melting point of the TLCP. Therefore, equilibrating the material at $310^{\circ} \mathrm{C}$ ensures all the crystalline structure is excluded. Upon cooling, the TLCP starts to crystallize at $232^{\circ} \mathrm{C}$, and the peak crystallization temperature is $228^{\circ} \mathrm{C}$. 


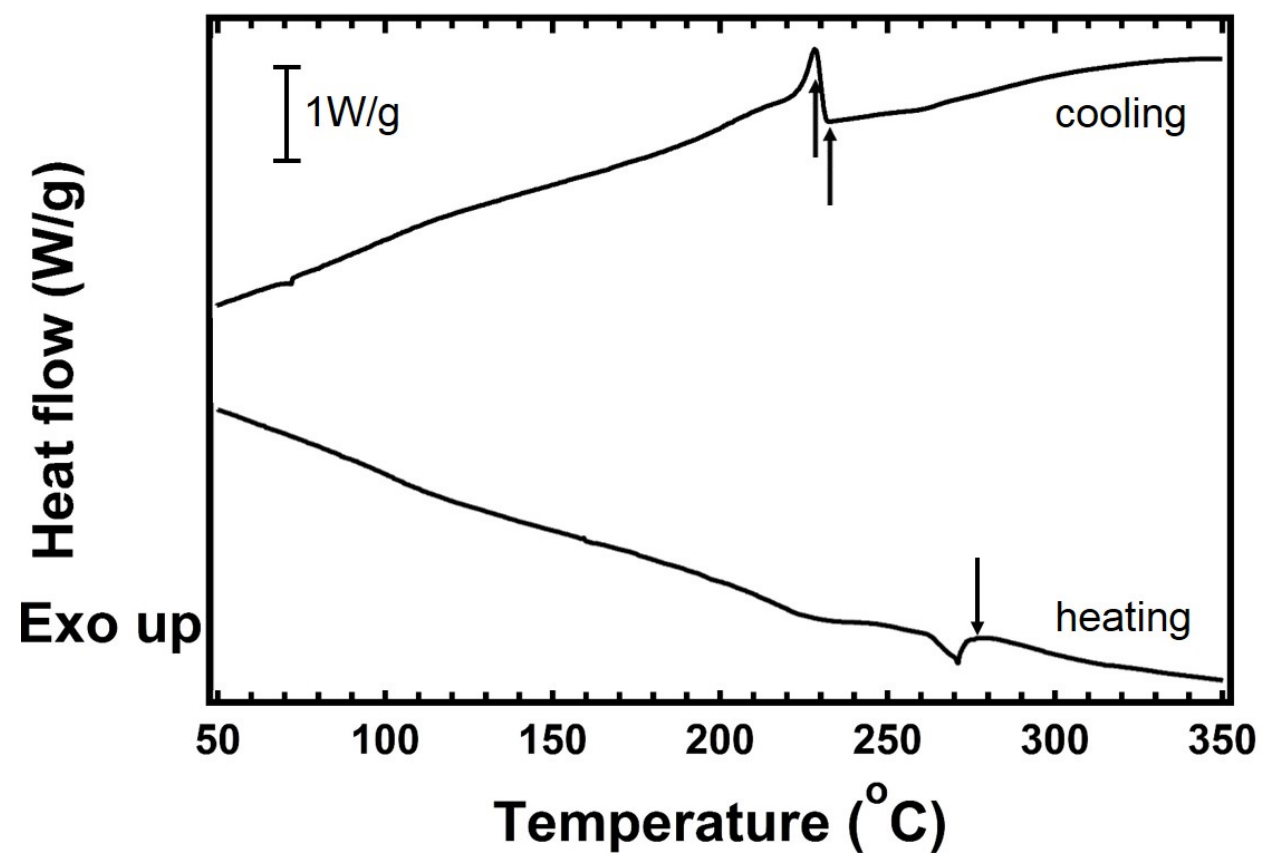

Figure 2: DSC heating and cooling scans of $\mathrm{HX} 8000$ at the rate of $20^{\circ} \mathrm{C} / \mathrm{min}$. The arrow pointing down indicates the melting point of the TLCP, and the arrows pointing up suggest the onset and peak crystallization temperatures, respectively. The vertical bar suggests the scale in heat flow.

Isothermal DSC is a commonly used technique to monitor the crystallization of TLCPs below their melting points [17]. After completely melted at $310^{\circ} \mathrm{C}$, the TLCP was cooled to one of the predetermined temperatures of 270,260 and $250^{\circ} \mathrm{C}$. The change in heat flow was recorded as a function of time, as shown in Fig 3. At all three given temperatures, DSC tests sense no heat released from the samples, which suggests isothermal crystallization does not occur at these temperatures. 


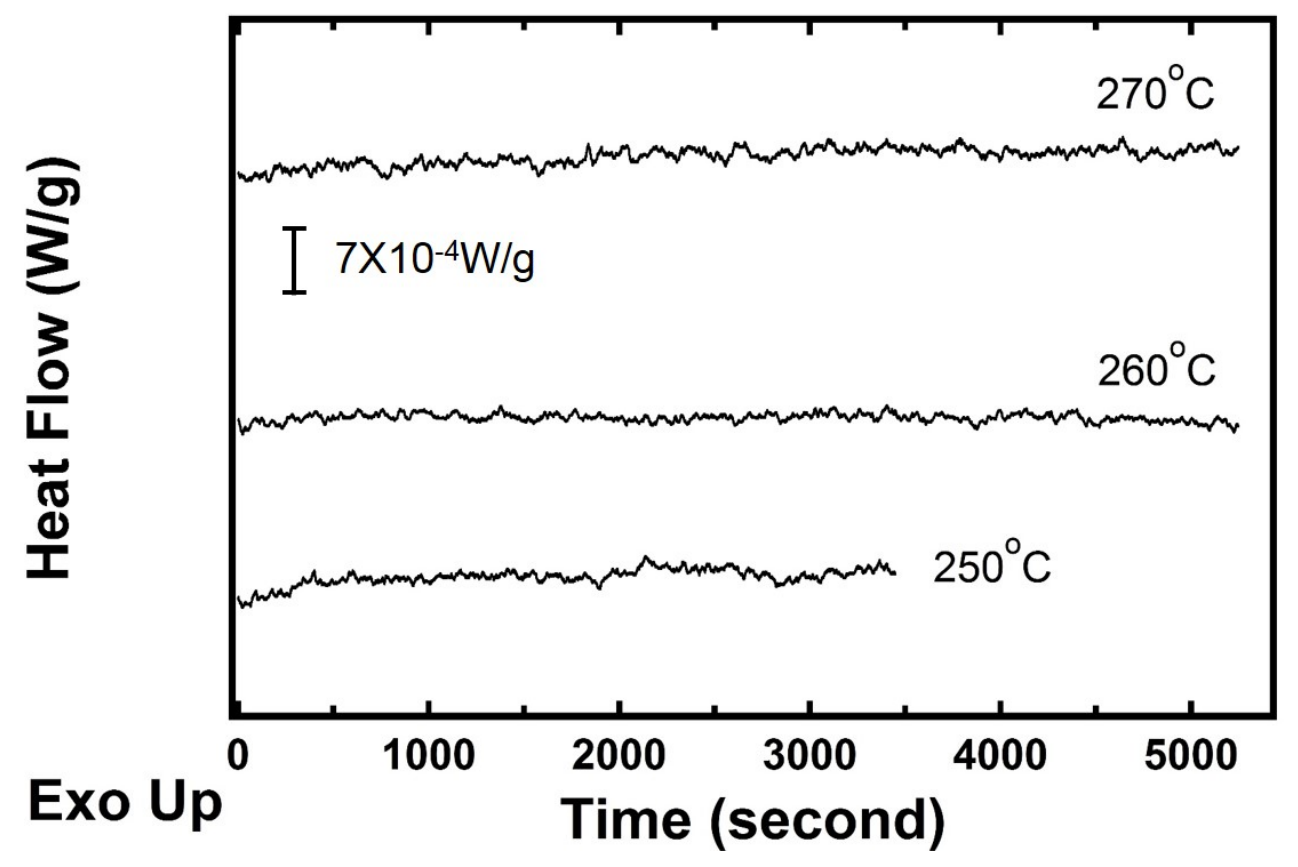

Figure 3: Isothermal DSC scans for $\mathrm{HX} 8000$ at 270,260 and $250^{\circ} \mathrm{C}, \mathrm{HX} 8000$ was cooled from $310^{\circ} \mathrm{C}$. The vertical bar suggests the scale in heat flow.

\subsection{Small amplitude oscillatory shear measurements}

To complement the crystallization temperature of the TLCP obtained from DSC, dynamic mechanical measurements in the temperature ramp mode were performed, as shown in Fig 4. Once melted, G' is greater than G' as the TLCP is cooled below its $280^{\circ} \mathrm{C}$ melting point, suggesting the material is still in the fluid state. Both G' and G" gradually increase as temperature decreases. At $235^{\circ} \mathrm{C}, \mathrm{G}^{\prime}$ surpasses G" and the crossover of G' and G' is observed. As reported in the literature, the crossover of G' and G' is a strong indication of TLCP crystallization [17]. Fig 4 shows the degree of supercooling is roughly $45^{\circ} \mathrm{C}\left(280\right.$ to $\left.235^{\circ} \mathrm{C}\right)$. Although it is hard to determine the exact time when the first crystal appears from SAOS results, in this report we take the crossover of G' and G" as a signature to indicate the early stage of crystallization. The crystallization temperature 
obtained from SAOS $\left(235^{\circ} \mathrm{C}\right)$ is in good agreement with that in the DSC test (onset crystallization temperature of $232^{\circ} \mathrm{C}$ ).

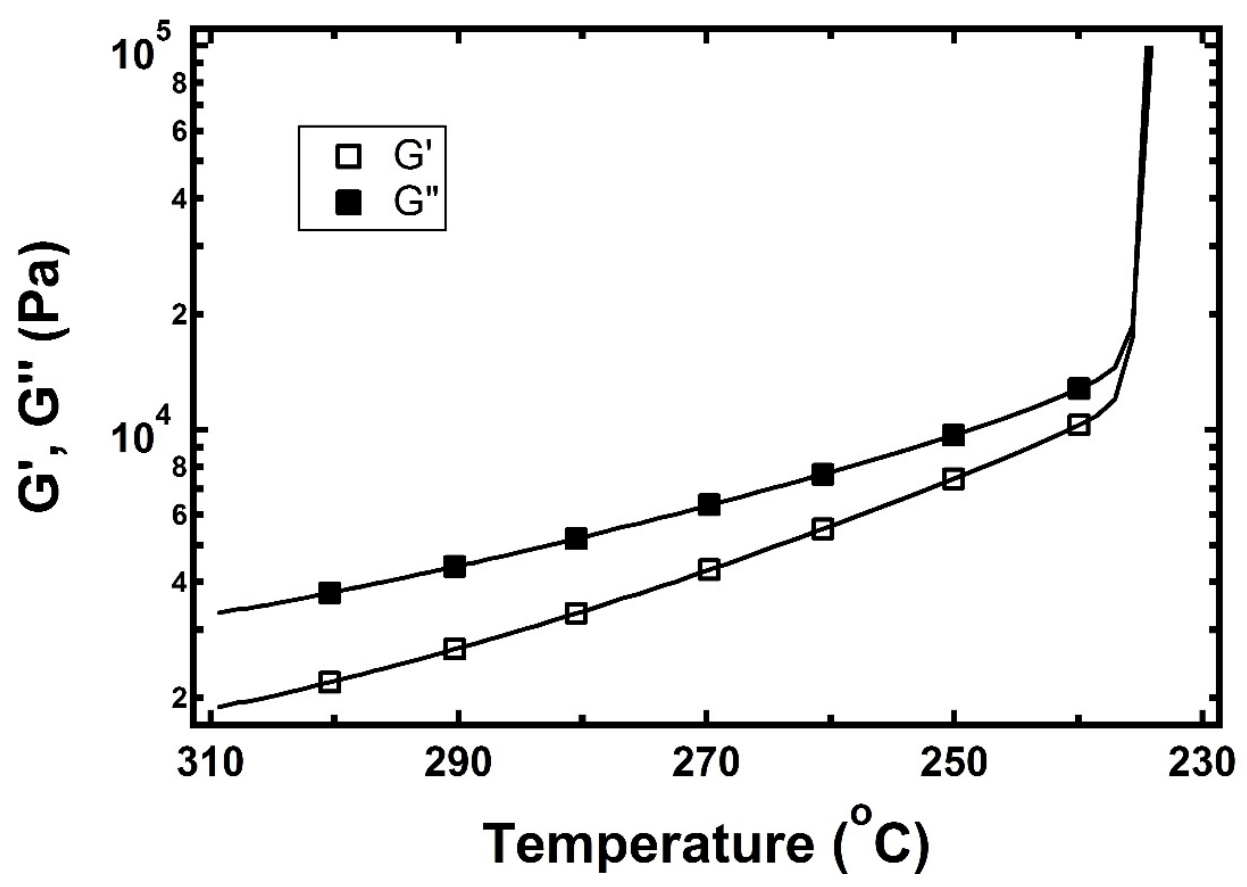

Figure 4: The storage modulus G' and loss modulus G” versus temperature as HX8000 was cooled at a rate of $20^{\circ} \mathrm{C} / \mathrm{min}$.

Meanwhile it is equally important to determine how long the TLCP could maintain fluidity at a given temperature in the super-cooled state. Therefore, dynamic rheological measurement was performed in the isothermal time sweep mode, and the result is shown in Figs 5. From the rheological test, it can be observed when the TLCP is cooled to 270 and $260^{\circ} \mathrm{C}, \mathrm{G}^{\prime}$ and $\mathrm{G}$ " are mostly parallel with one another and no crossover can be identified within the extended test period (1.5 hour). At these two temperatures the TLCP is relatively stable. On the other hand, crystallization occurs at $250^{\circ} \mathrm{C}$ as $\mathrm{G}^{\prime}$ and G" display a crossover 2550 s after the measurement starts. The slow crystallization rate of the TLCP at this temperature indicates this temperature in the super-cooled state can also be used for rheological tests. 


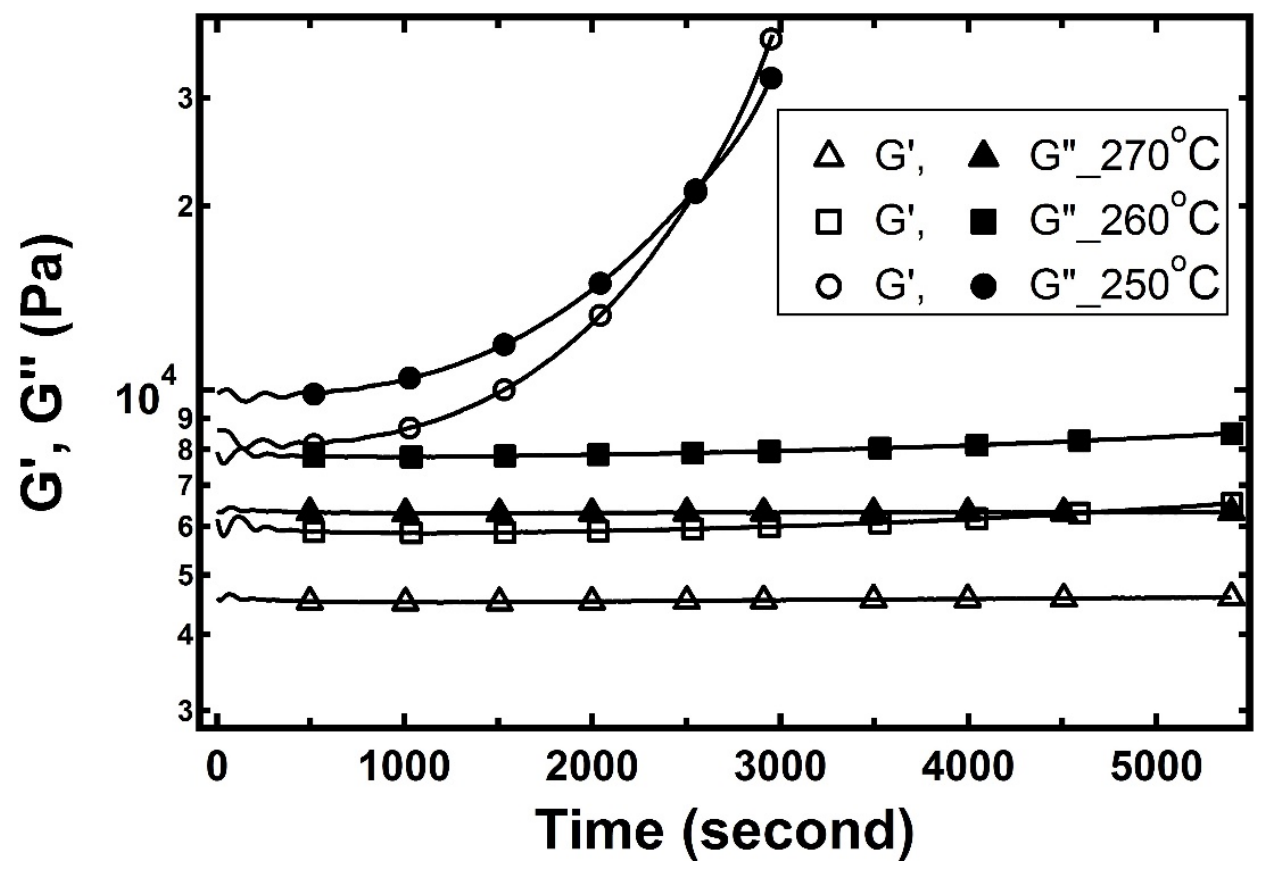

Figure 5: The storage modulus G' and loss modulus G" versus time as HX8000 was cooled to different temperatures below $\mathrm{T}_{\mathrm{m}}$.

Both SAOS and DSC suggest crystallization does not occur when the TLCP melt is cooled to 270 and $260^{\circ} \mathrm{C}$. However, the discrepancy between DSC and rheological results is observed at $250^{\circ} \mathrm{C}$. SAOS indicates crystallization occurs at this temperature, but DSC does not. The discrepancy is due to the two possible reasons. First, shear-induced crystallization may only occur in the SAOS measurements. The strain applied in the measurement could align the molecules and accelerate crystallization. However, we believe this is not the case. In another study we tested the crystallization of a TLCP of $60 \mathrm{~mol} \%$ hydroxynaphthoic acid, $20 \mathrm{~mol} \%$ terephthalic acid and 20 mol\% 4-aminophenol in the super-cooled state, using SAOS with 1, 2.5 and 5\% strain, respectively [36]. If shear-induced crystallization did occur, it should take less time for G' to surpass G" as strain increases. Nevertheless, the crossover time of G' and G' was independent of the amount of strain applied, which suggested shear-induced crystallization did not occur at $2.5 \%$ strain. Another explanation is that DSC may not possess sufficient sensitivity for detecting the onset of 
crystallization. That is, the initial stage of TLCP crystallization may only release a traceable amount of heat, which is not detectable by DSC. Lin and Winter [37] showed DSC results were not sensitive enough to pick up the melting transition of residual 73/27 HBA/HNA crystals in the heating scan. In contrast, the detection for the onset of crystallization in SAOS relies on the influence of TLCP crystals on the flow behavior of the polymer melt, which is independent of the heat change during the phase transition. Therefore, we suggest the rheological measurement is a more effective method in sensing the onset of TLCP crystallization in the super-cooled state.

\subsection{Startup of shear flow}

To complement the results obtained from small amplitude oscillatory shear measurements, transient shear in the startup of shear flow was carried out. Liquid crystalline polymers are known to be unusually susceptible to the edge fracture instability in steady shear flow when being tested by rotational rheometers with the cone-and-plate fixtures [38-39]. Only relatively low shear rates are applied to avoid the occurance of edge fracture. In our work as the TLCP was cooled to the super-cooled state, it became higly elastic, as indicated by the increase in G' in Fig 4. The high elasticity of the material made measuring the transient rheology a challenging task. No reliable results can be obtained using the conventional cone-and-plate fixtures because of inevitable edge fracture. Baek et al. [38-39] suggested using fixtures with narrow gaps at the rim to delay edge fracture at higher shear rates. This approach was adopted in our work and parallel disks fixtures with $0.2 \mathrm{~mm}$ gap were used. Even so the maximum shear rate achieved was only $0.5 \mathrm{~s}^{-1}$ in the super-cooled state. The cone-and-plate fixtures are usually preferrable for transient rheological measurments because of the uniform shear rate across the radius. Therefore, a comparison between the results obtained from these two fixtures is necessary to ascertain whether at least qualitative agreement can be achieved. The comparison of stress growth curves in flow inception at $310{ }^{\circ} \mathrm{C}$ 
and $0.5 \mathrm{~s}^{-1}$ is shown in Fig 6 . The $0.5 \mathrm{~s}^{-1}$ shear rate corresponds to the shear rate at the edge for the parallel disks fixtures, and it is uniform across the radius for the cone-and-plate fixtures. Although quantitatively the two curves do not match completely, parallel disks capture the same features of transient shear stress behavior as those obtained from the cone-and-plate. Both curves exhibit an initial overshoot, followed by an undershoot and a second overshoot. Additionally, when the material is sheared to a steady state, the values of both shear stresses are quite close. Thus, transient measurements using parallel disks allow at least a qualitative investigation into the effect of temperature on the shear induced structure evolution of the TLCP.

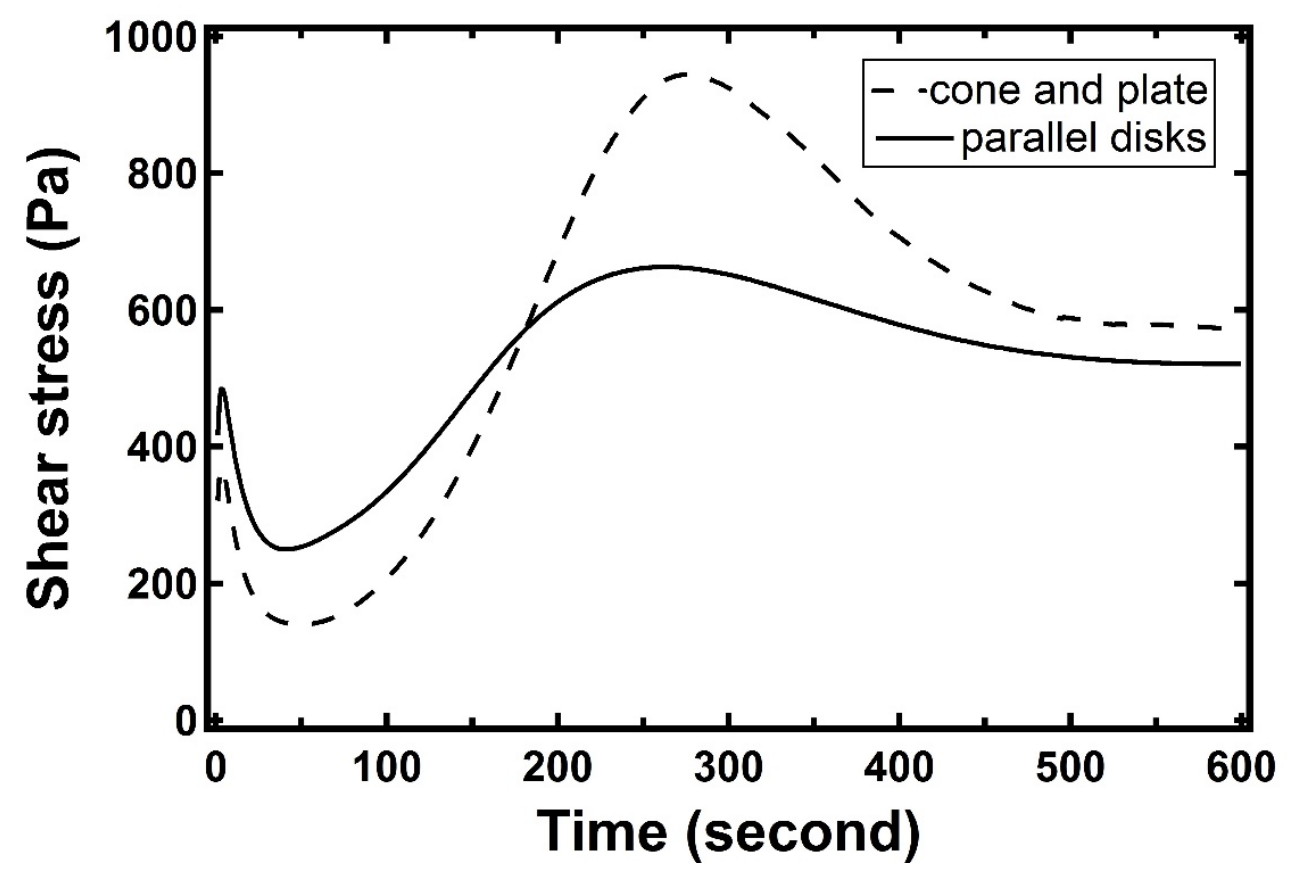

Figure 6: Transient stress growth of $\mathrm{HX} 8000$ in flow incpetion at $310^{\circ} \mathrm{C}$ and $0.5 \mathrm{~s}^{-1}$, using coneand-plate ( $0.1 \mathrm{rad}, 25 \mathrm{~mm}$ diameter) and parallel disks fixtures (25 $\mathrm{mm}$ diameter), respectively.

Although a negative first normal stress difference, $N_{1}$, was frequently observed for lyotropic liquid crystalline polymers, and the mechanism was well understood [40], the sign of $N_{1}$ was still under debate for TLCPs. Guskey and Winter [28] reported negative $N_{1}$ values at low shear 
rates for the 73/27 HBA/HNA. In contrast Cocchini et al. [25] reported all positive $N_{1}$ values over the shear rate range from 0.02 to $10 \mathrm{~s}^{-1}$. Cocchini et al. explained the discrepancy by demonstrating the long decay of the normal stress after sample loading/squeezing procedures and the significant residual normal stress at the startup of shear flow. If the residual stress experienced by the transducer at the beginning of flow inception was taken as the "zero level stress", during the transient experiments, the continuous decay of the normal stress led to erroneous interpretations of the negative $N_{1}$ values. Therefore, previous studies emphasized the importance of starting the transient measurements with negligible normal stress $(<10 \mathrm{~g})$ [24-25]. On the other hand, in our study parallel disks fixtures with $0.2 \mathrm{~mm}$ gap were chosen to avoid edge fracture. The small gap resulted in a significant amount of normal stress $(\sim 150 \mathrm{~g})$ during sample loading. Even after $1 \mathrm{hr}$ of relaxation, the amount of residual normal stress was till substantial $(\sim 50 \mathrm{~g})$. Due to the thermal stability of the liquid crystalline polyester, waiting for the normal stress to decay completely was impractical. As a consequence, the $N_{1}$ recorded did not truly reflect the material response, because it was also influenced by the decay of residual normal stress. Therefore the unreliable $N_{1}$ results are not shown and discussed in the paper.

Figs 7 and 8 show the respective growth of the transient shear stress or viscosity as a function of time in the flow inception experiments at shear rates of 0.1 and $0.5 \mathrm{~s}^{-1}$. Due to edge fracture, the highest achievable shear rate was $0.5 \mathrm{~s}^{-1}$ in the super-cooled state. On the other hand, $0.1 \mathrm{~s}^{-1}$ was the lowest shear rate can be applied in the super-cooled state. It took more than $1 \mathrm{hr}$ to reach the steady state at lower shear rate, and long testing time caused thermal stability problems, such as offgassing [41]. In Figs 7 and 8, the steady state viscosity of the TLCP is significantly enhanced when it is cooled to the super-cooled state. For example, when the temperature is lowered 
from 310 to $250{ }^{\circ} \mathrm{C}$, the viscosity at 0.1 and $0.5 \mathrm{~s}^{-1}$ is increased from $1180 \mathrm{~Pa} \cdot \mathrm{s}^{-1}$ to $11,000 \mathrm{~Pa} \cdot \mathrm{s}^{-1}$

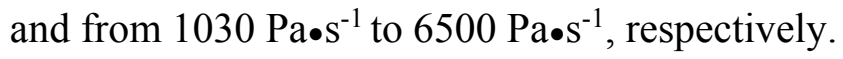

Additionally, when the TLCP is super cooled below its melting point, it is interesting that the shapes of the stress growth curves in the super-cooled state are qualitatively similar to that at $310^{\circ} \mathrm{C}$. Previous work shows two physical phenomena are potentially responsible for the complicated stress behavior. One is the reorientation of the rod-like molecules [18] and the other is the rearrangement of the domain structure [27]. The similarity in the shapes of the curves suggests the transient rheological behavior is caused by the same type of structure change, both at $310^{\circ} \mathrm{C}$ and in the super-cooled state. However, it is difficult to assign the exact cause for each overshoot as well as the undershoot solely based on the transient rheological measurements. It could be the orientation of molecules, or the rearrangement of the domain structure, or the combination of both. Our ongoing efforts include employing direct structure characterizations to further elucidate the complex transient stress behavior. The two important methods are the in situ X-ray scattering [29] and the rheo-optical technique [21], which provide information on the orientation dynamics of the molecules and the rearrangement of the domain structure, respectively. Combining the structure evolution and the transient stress response, the overshoots and undershoot in Figs 7 and 8 can be better understood. For example, upon shearing, if the molecules already reorientate to a steady state before an overshoot occurs, while the domain structure is still evolving, then the domain structure rearrangement is the cause for the overshoot, and vice versa.

It should also be noticed as the temperature is lowered, the location of the stress overshoots and undershoots is shifted to the right along the time axis, and the detailed location of the peaks is shown in Table 1. The shift of the peak location indicates the structure evolution that causes the stress growth is slowed down when the TLCP is cooled down to the super-cooled state. 
At $250^{\circ} \mathrm{C}$, it takes about the same amount of time (2400 s) before both stress growth curves at 0.1 and $0.5 \mathrm{~s}^{-1}$ exhibit a continuous increase. In the dynamic mechanical measurements in Fig 5, the crossover of G' and G" also occurs at $2550 \mathrm{~s}$. Therefore, the continuous increase in transient shear stress at $250^{\circ} \mathrm{C}$ after $2400 \mathrm{~s}$ is attributed to the crystallization of HX8000. When crystallization occurs, the total amount of strain $(\dot{\gamma} \mathrm{t})$ applied to the TLCP in the startup of shear flow at 0.1 and $0.5 \mathrm{~s}^{-1}$ is 240 and 1200 strain units, respectively. Because the crystallization rates at $250{ }^{\circ} \mathrm{C}$ at these two shear rates are comparable and independent of strain, we believe shearinduced crystallization does not occur at this temperature. Otherwise the stress at $0.5 \mathrm{~s}^{-1}$ should grow faster than that at $0.1 \mathrm{~s}^{-1}$.

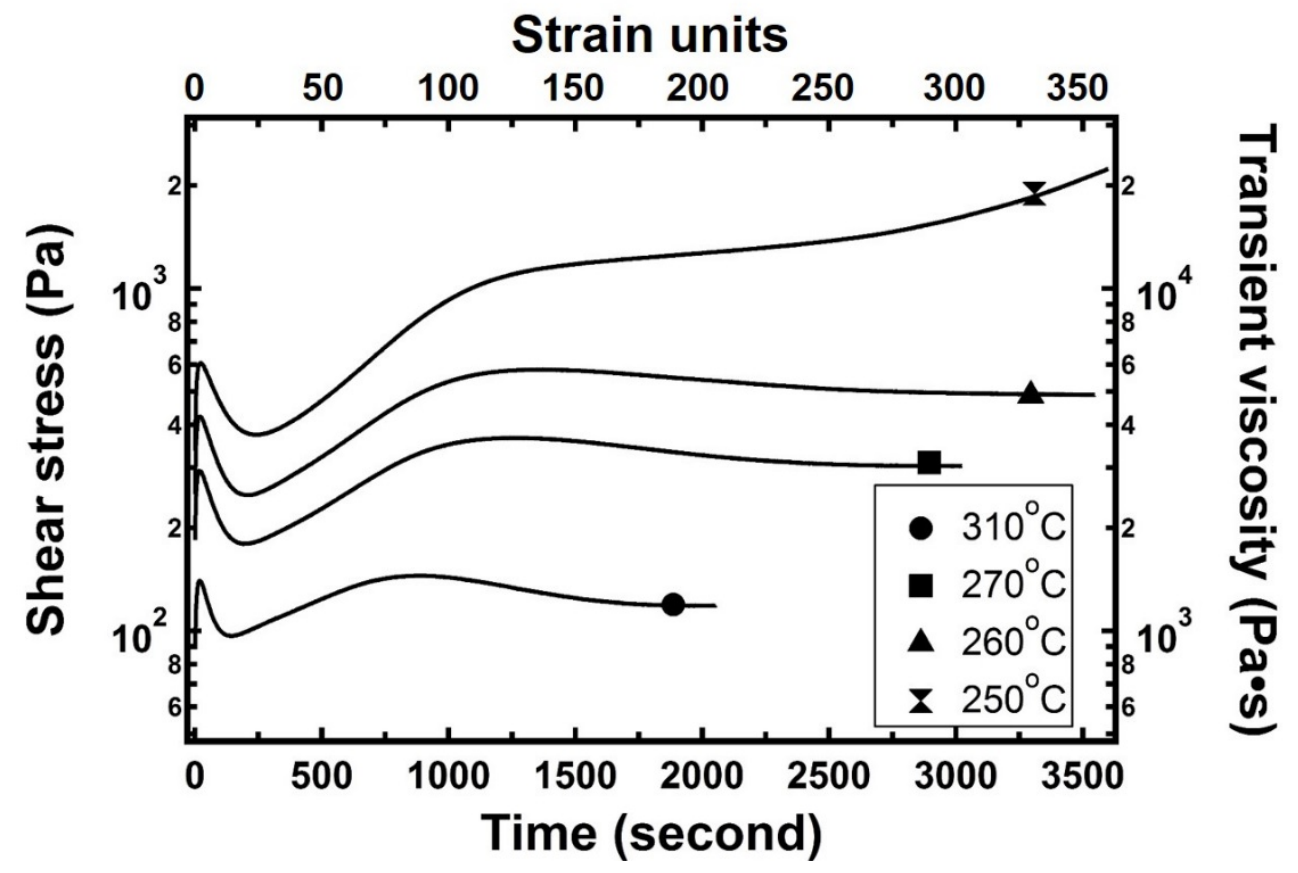

Figure 7: Growth curves of transient shear stress and viscosity above the melting point $\left(310^{\circ} \mathrm{C}\right)$ and in the super-cooled state $\left(270,260\right.$ and $\left.250^{\circ} \mathrm{C}\right)$ for HX8000. Shear rate equals $0.1 \mathrm{~s}^{-1}$. 


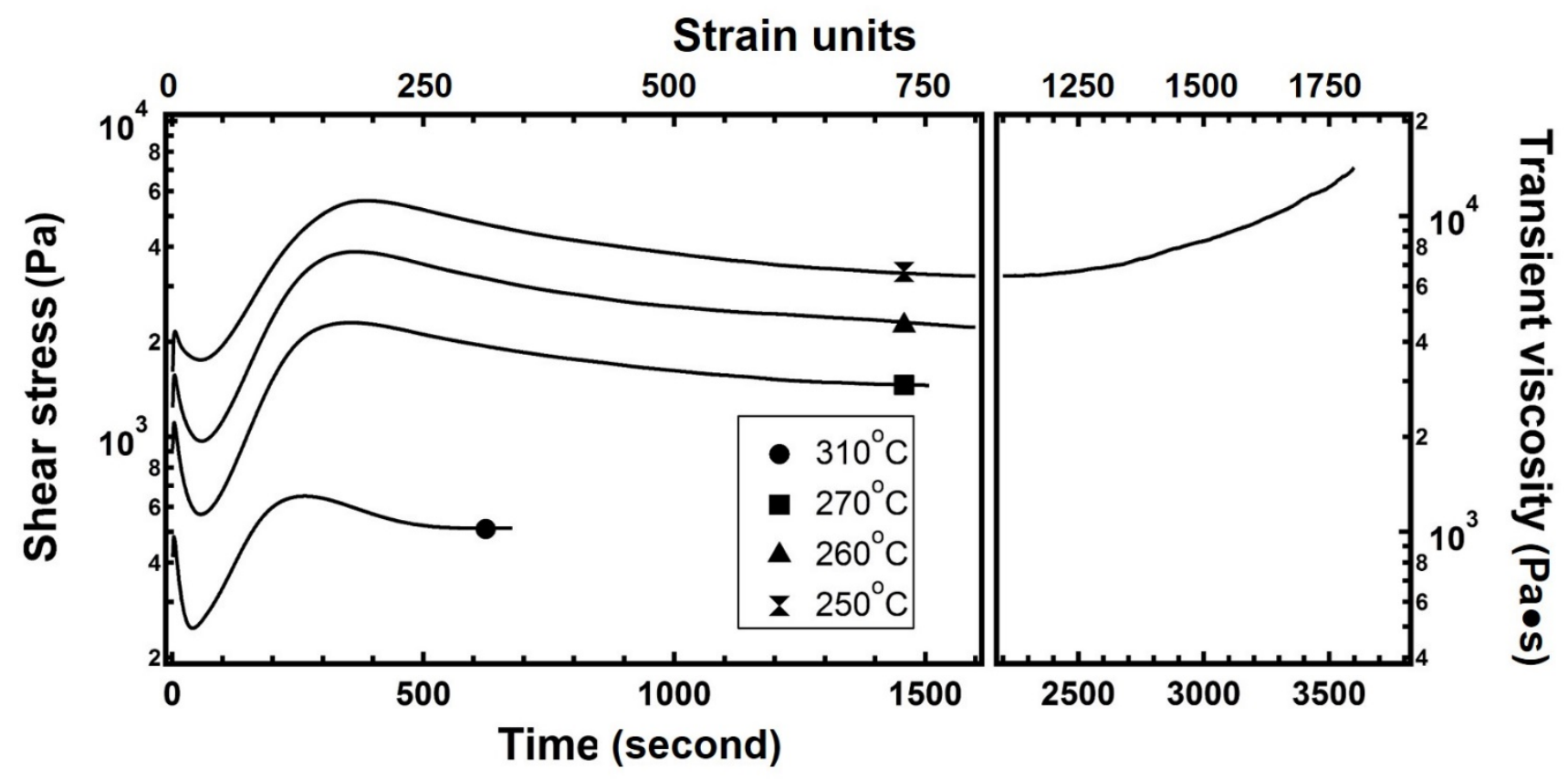

Figure 8: Growth curves of transient shear stress and viscosity above the melting point $\left(310^{\circ} \mathrm{C}\right)$ and in the super-cooled state $\left(270,260\right.$ and $\left.250^{\circ} \mathrm{C}\right)$ for $\mathrm{HX} 8000$. Shear rate equals $0.5 \mathrm{~s}^{-1}$.

Table 1: Locations the overshoots and undershoot of shear stress in time units at $310^{\circ} \mathrm{C}$ (above the melting point) and in the super-cooled state $\left(270,260\right.$ and $\left.250^{\circ} \mathrm{C}\right)$.

\begin{tabular}{|c|c|c|c|c|}
\hline \multirow{3}{*}{ Shear rate $/ \mathrm{s}^{-1}$} & \multirow{2}{*}{ Temperature $/{ }^{\circ} \mathrm{C}$} & \multicolumn{3}{|c|}{ Peak position on time axis /s } \\
\cline { 3 - 5 } & & Initial overshoot & undershoot & $\begin{array}{c}\text { Second } \\
\text { overshoot }\end{array}$ \\
\hline \multirow{3}{*}{0.1} & 310 & 18 & 146 & 861 \\
\cline { 2 - 5 } & 270 & 19 & 197 & 1277 \\
\cline { 2 - 5 } & 260 & 21 & 210 & 1373 \\
\cline { 2 - 5 } & 250 & 23 & 240 & NA \\
\hline \multirow{3}{*}{0.5} & 310 & 3 & 40 & 333 \\
\cline { 2 - 5 } & 270 & 4 & 58 & 357 \\
\cline { 2 - 5 } & 260 & 5 & 62 & 394 \\
\hline
\end{tabular}




\subsection{Relaxation after flow cessation}

Fig 9 shows the relaxation of shear stress after $1600 \mathrm{~s}$ preshearing at $0.5 \mathrm{~s}^{-1}$. At $310^{\circ} \mathrm{C}$, the relaxation of shear stress consists a fast initial drop and a much slower decay. Interestingly enough, after being sheared in the super-cooled state, all the shear stress relaxation curves contain both the fast and slow decay regions as well, similar to that at $310^{\circ} \mathrm{C}$. Even in the super-cooled state, most of the residual shear stress eventually dies out.

The relaxing shear stress at different temperatures is also normalized with respect to the steady states value obtained in preshearing, and the normalized shear stress is plotted as a function

of time in Fig 10. The initial fast decay part of the curves overlaps really well for different temperatures in the super-cooled state, and the curves obtained in the super-cooled state are parallel with that above the melting point. This part of each curve is expected to be associated with the rearrangement of the molecules, for example the relaxation of molecular orientation [42]. Thus it seems cooling the material to the super-cooled state does not significantly altered the orientation relaxation of the molecules. 


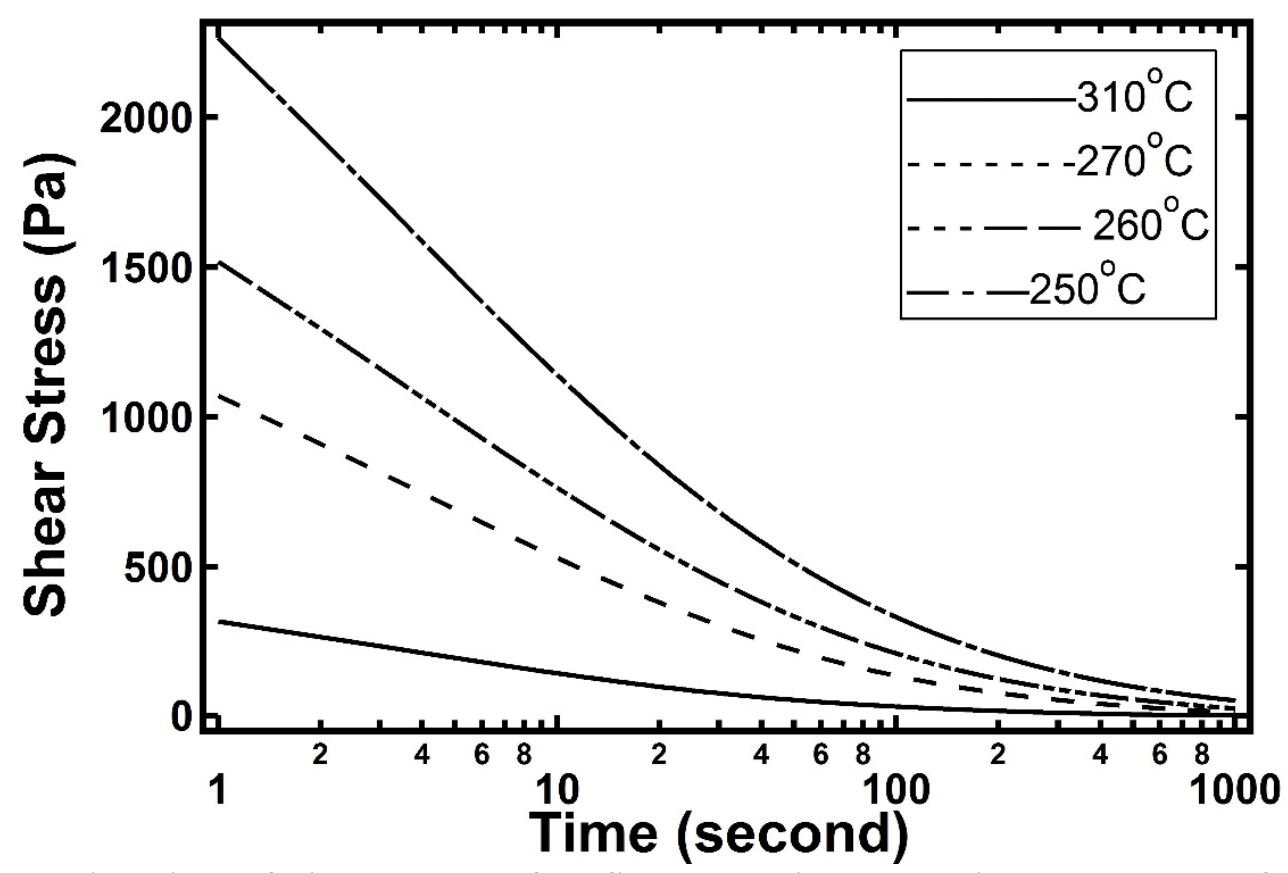

Figure 9: Relaxation of shear stress after flow cessation. Experiments were performed at the temperature of $310^{\circ} \mathrm{C}$ and in the super-cooled state $\left(270,260\right.$ and $\left.250^{\circ} \mathrm{C}\right)$ for the TLCP.

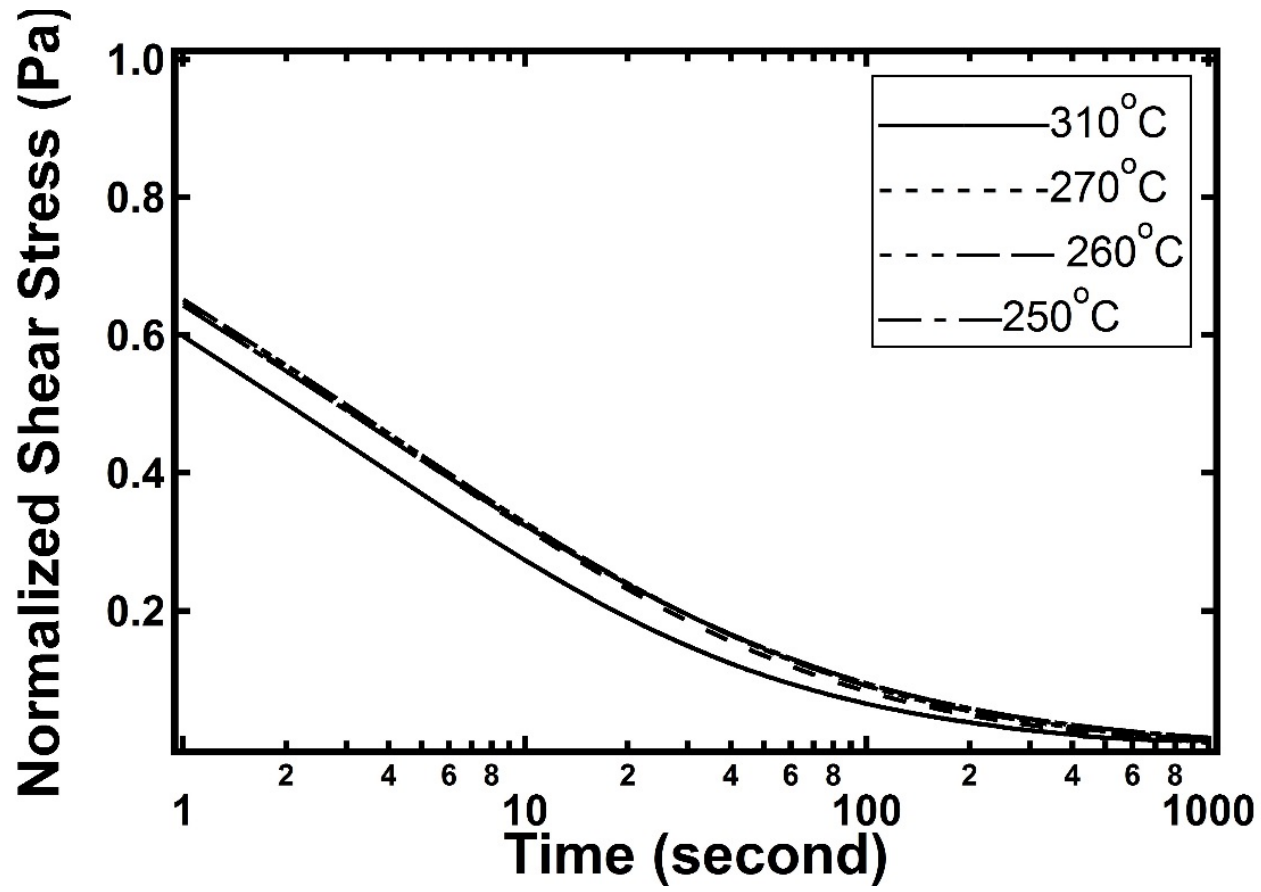

Figure 10: Relaxation of shear stress after flow cessation. Experiments were performed at the temperature of $310^{\circ} \mathrm{C}$ and in the super-cooled state $\left(270,260\right.$ and $\left.250^{\circ} \mathrm{C}\right)$ for the TLCP. The shear stress was normalized with respect to the steady state value obtained in preshearing. 


\subsection{Small amplitude oscillatory shear after flow cessation}

Applying small amplitude oscillatory shear immediately after flow cessation has been a convenient approach to probe the structure evolution during relaxation. In this method a decrease in loss modulus, G", suggests an increase in the degree of molecular orientation and vice versa. Here we also adopted this indirect method to track the evolution of molecular orientation after shearing at 310,270 and $260^{\circ} \mathrm{C}$. After the TLCP was sheared at $0.5 \mathrm{~s}^{-1}$ for $1600 \mathrm{~s}$, small amplitude oscillatory shear with $2.5 \%$ strain and $10 \mathrm{rad} / \mathrm{s}$ frequency was imposed for $600 \mathrm{~s}$. The change in G" as a function of time is recorded in Fig 11 . At $310^{\circ} \mathrm{C}, \mathrm{G}$ ” keeps increasing, indicating the relaxation of shear-induced orientation.

Before we analyze the change of G" in the super-cooled state and relate that to the molecular orientation evolution, it is important to ascertain whether the change of G" is caused by the relaxation of molecular orientation instead of the crystallization of the TLCP. Based on the crystallization kinetics study in Fig 5, the change of G” is negligible at 270 and $260^{\circ} \mathrm{C}$. Consequently, the apparent increase in G" in Fig 11 at these two temperatures could only be attributed to the relaxation of molecular orientation. In contrast, at $250^{\circ} \mathrm{C}$, the change in G" after preshearing will not be only due to the molecular orientation evolution, it will also be greatly influenced by crystallization, as suggested by Fig 5 . Thus the result at $250^{\circ} \mathrm{C}$ will not be shown.

When the TLCP is cooled to 270 and $260^{\circ} \mathrm{C}$, G" gradually increases as time elapses. One critical finding from Fig 11 is the curves at all three temperatures are almost parallel with one another. This serves as further evidence that super cooling the TLCP below its melting point probably does not influence the orientation dynamics of the molecules significantly. 


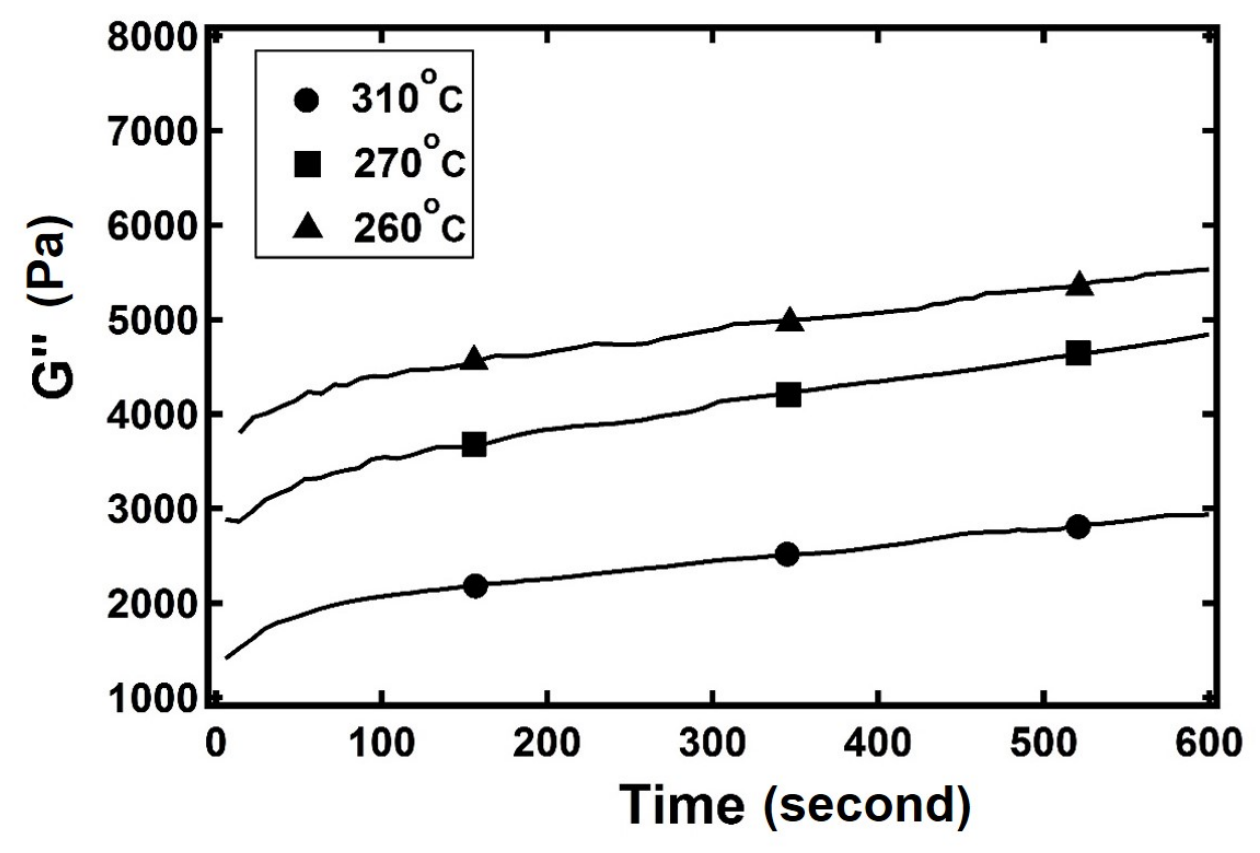

Figure 11: Evolution of G" after flow at $0.5 \mathrm{~s}^{-1}$ for $1600 \mathrm{~s}$ for the TLCP. Experiments were performed at the temperature of $310^{\circ} \mathrm{C}$ and in the super-cooled state $\left(270,260^{\circ} \mathrm{C}\right)$. The strain and frequency used were $1.0 \%$ and $10 \mathrm{rad} / \mathrm{s}$, respectively.

\subsection{Interrupted shear}

The structure recovery after flow cessation was investigated by interrupted shear experiments at temperatures both above the melting point and in the super-cooled state. In this set of tests after the TLCP was sheared to steady state, the material was allowed to relax for 50, 200 and 500s and flow was resumed. The stress growth after flow restarted at $310^{\circ} \mathrm{C}$ is presented in Fig 12. To better elucidate the recovery of transient stress as a function of relaxation time, the shear stress is normalized by dividing by the steady state value. The shear stress growth obtained in the startup of shear flow is also presented as a reference. With the use of a longer relaxation time, the magnitude of both undershoot and second overshoot is increased. The key observation in Fig 12 is the longer the TLCP is allowed to relax, the more similar the transient stress behavior upon flow resumption is relative to the initial curve in the flow inception. This is because of the 
structure recovery of the material. When flow is turned off, the average degree of orientation decrease, as suggested by Fig 11. Meanwhile it is highly possible that the domain structure disturbed in the startup of shear flow can recover its original status, before shearing is applied. For example, Guo et al. [21] directly observed the deformation of domains and reduction in domain size upon shearing using a rheo-optical technique. Although it may take a relative long time, the domain structure did recover to a certain degree after flow stopped.

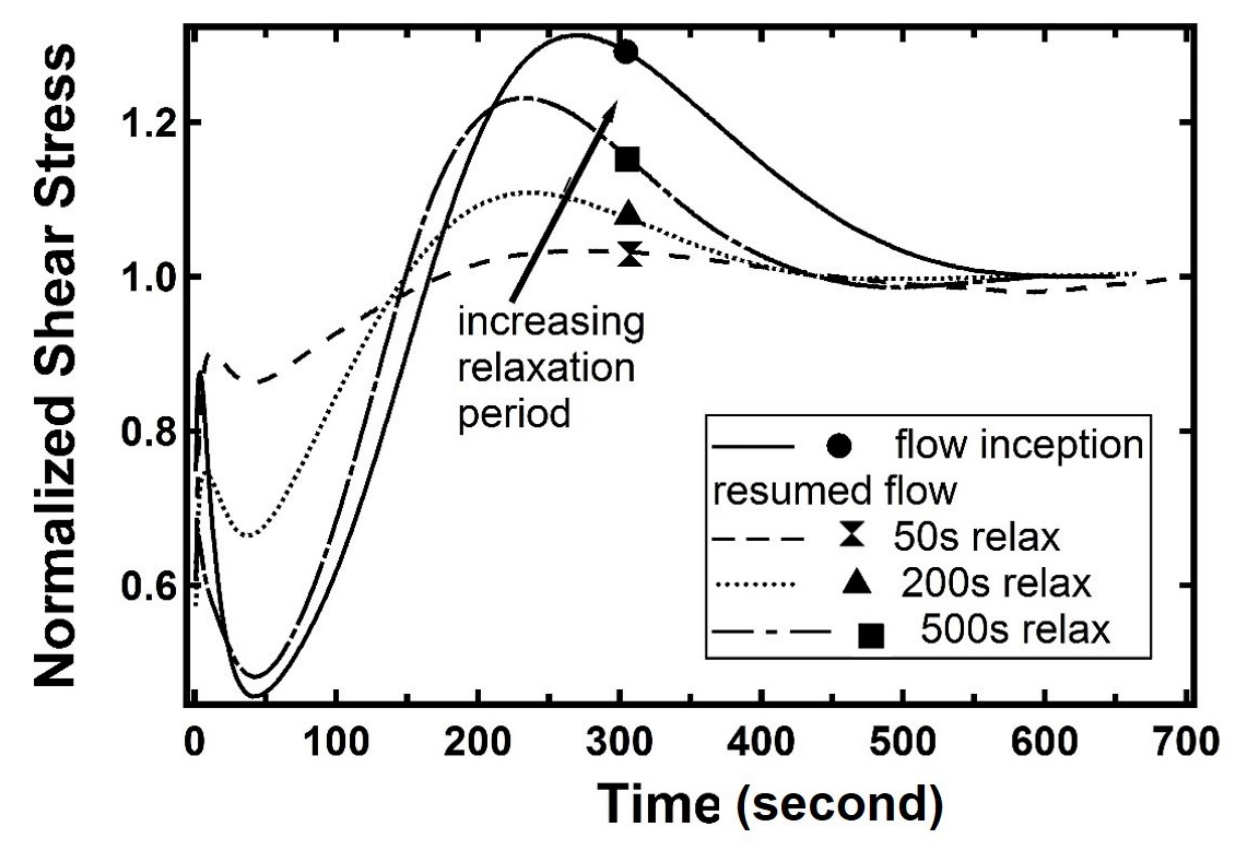

Figure 12: Trace of normalized transient shear stress in interrupted shear after relaxaing for 50 , 200 and $500 \mathrm{~s}$ at $310^{\circ} \mathrm{C}$. The stress growth in the startup of shear flow is also shown as a reference.

Now we turn our attention to the transient stress growth after relaxation in the super-cooled state. Figs 13 to 15 show the transient stress behavior at the temperatures of 310,270 and $260^{\circ} \mathrm{C}$, after relaxing for 50, 200 and 500s. Although the absolute value of stress increases as temperature is lowered, each curve is divided by the steady state stress, such that the influence of temperature on the transient stress behavior could be better visualized. As discussed earlier, the longer the relaxation time, the higher the degree of recovery in the transient shear stress is observed at $310^{\circ} \mathrm{C}$. 
However, this is not the case for the temperatures of 270 and $260^{\circ} \mathrm{C}$. Even after $500 \mathrm{~s}$ relaxation, stress recovery at 270 and $260^{\circ} \mathrm{C}$ can be barely observed.

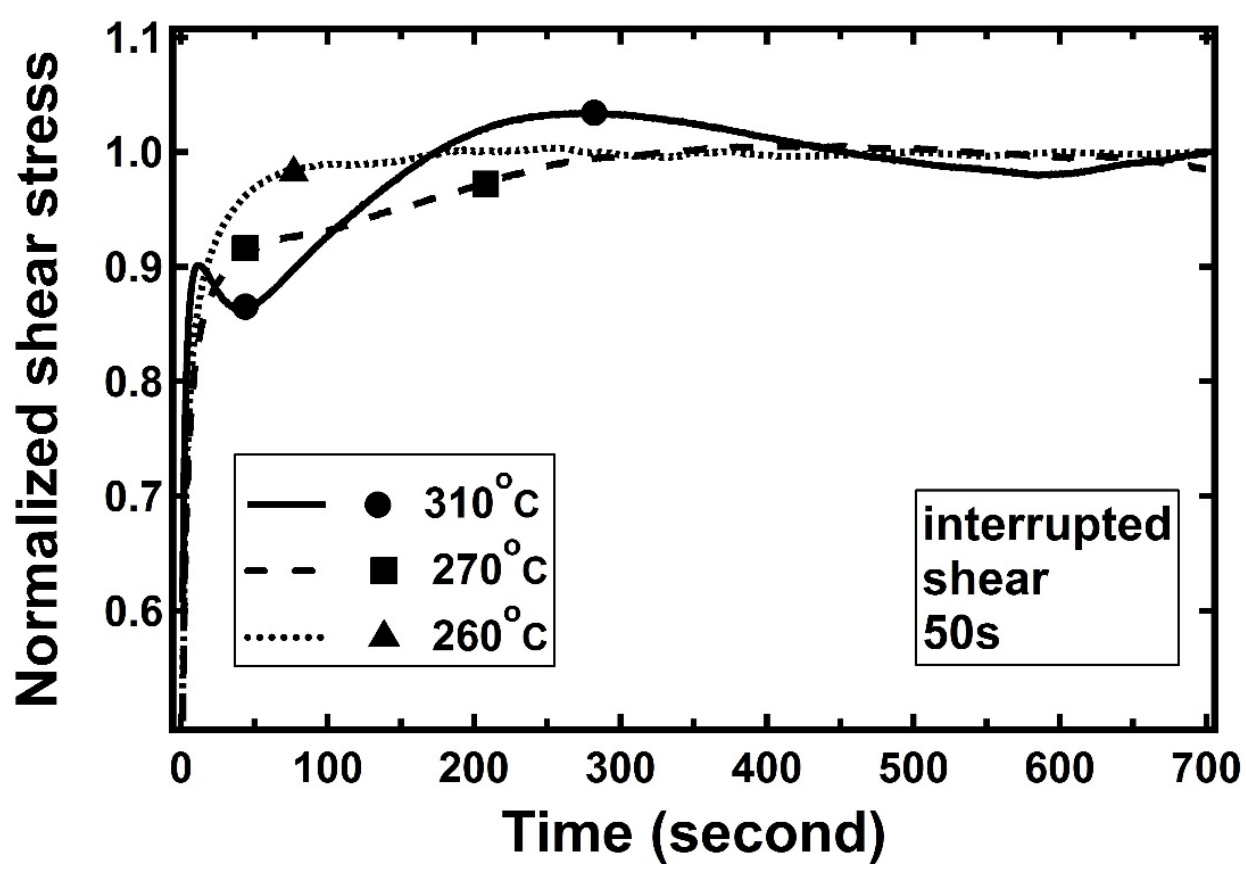

Figure 13: Trace of normalized transient shear stress in interrupted shear after 50s relaxation. Measurements were performed at 310,270 and $260^{\circ} \mathrm{C}$. 


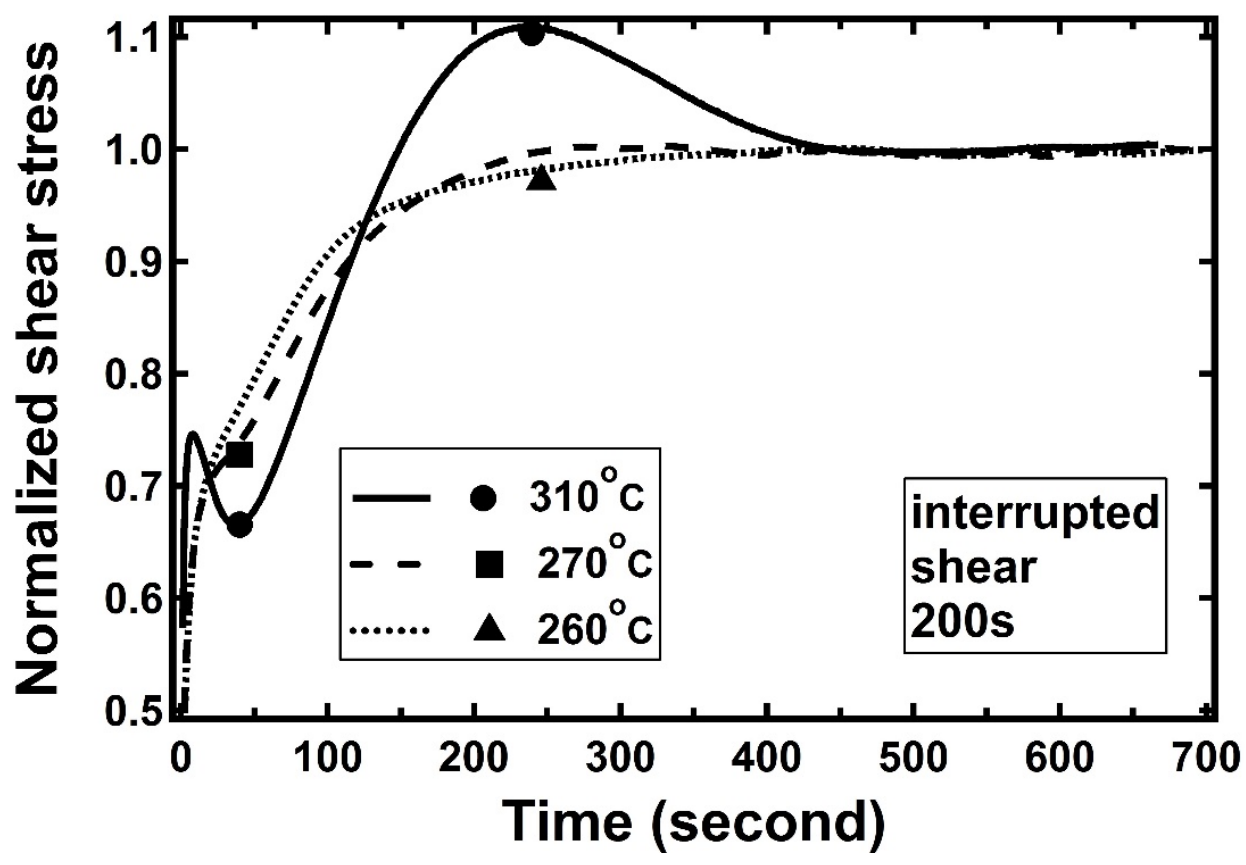

Figure 14: Trace of normalized transient shear stress in interrupted shear after 200s relaxation. Measurements were performed at 310,270 and $260^{\circ} \mathrm{C}$.

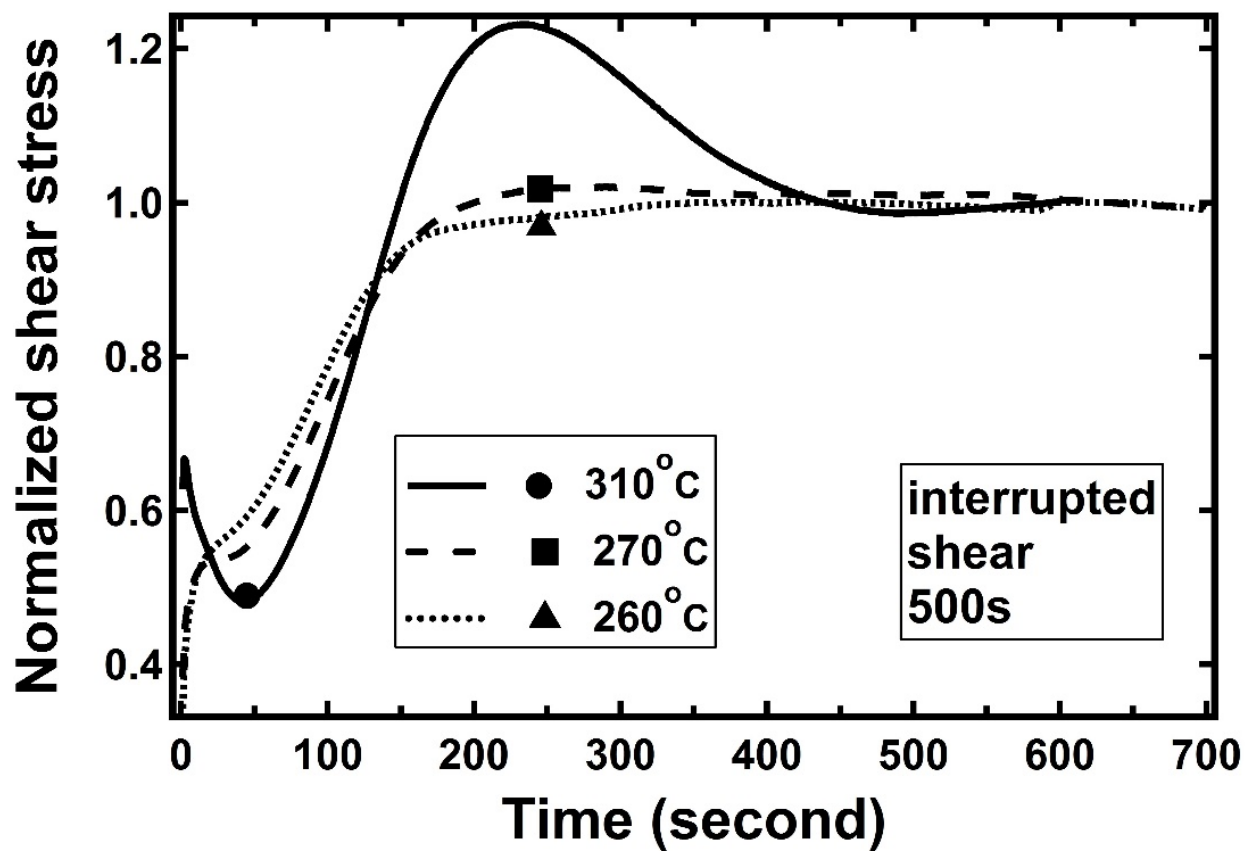

Figure 15: Trace of normalized transient shear stress in interrupted shear after 500s relaxation. Measurements were performed at 310,270 and $260^{\circ} \mathrm{C}$. 
The unusual low degree of transient stress recovery in the super-cooled state indicates the structure evolution is slowed down significantly after flow inception. Figs 10 and 11 suggest lowering the temperature to the super-cooled state surprisingly does not greatly alter the relaxation of the average molecular orientation when flow is stopped. Consequently, the reorientation of the TLCP molecules should not be the reason for the complicated transient stress behavior. Otherwise gradual recovery in shear stress should be observed at 270 and $260^{\circ} \mathrm{C}$. As we exclude the orientation evolution to be the cause for the transient stress behavior, it seems the rearrangement in the domain structure is solely responsible for the transient shear stress growth. Direct structure characterizations, such as in situ X-ray scattering [29] and rheo-optical measurement [21], will be helpful to confirm our interpretation and further elucidate the complicated transient stress response. The evolution in domain structure is probably slowed down as the TLCP is cooled to the supercooled state. Although we did not assess the change in domain structure and size directly by using rheo-optical methods, our interpretation is in good agreement with that from the study on $40 / 60$ PET/HBA [27].

\subsection{Flow reversal}

The reverse transient flow results are presented in Fig 16 to 19. Similar to Fig 13 to 15 , the transient shear stress is expressed as the normalized stress. In Fig 16, flow reversal is directly applied after 1600s of preshearing, and hardly any transient stress behavior can be observed at the temperatures both above the melting point and in the super-cooled state. Similar to the interrupted shear results in Fig 13 to 15, as the relaxation time increases, the transient shear stress response recovers to a greater extent relative to that in the startup of shear flow above the melting point. Nevertheless, there is still negligible transient stress recovery in the super-cooled state even after $500 \mathrm{~s}$ relaxation. The results in Fig 16 to 19 are in good agreement comparing to those obtained 
from the interrupted shear measurement. As a result, the flow reversal tests support our interpretation that the structure recovery of the TLCP is slowed down to a great extent in the supercooled state.

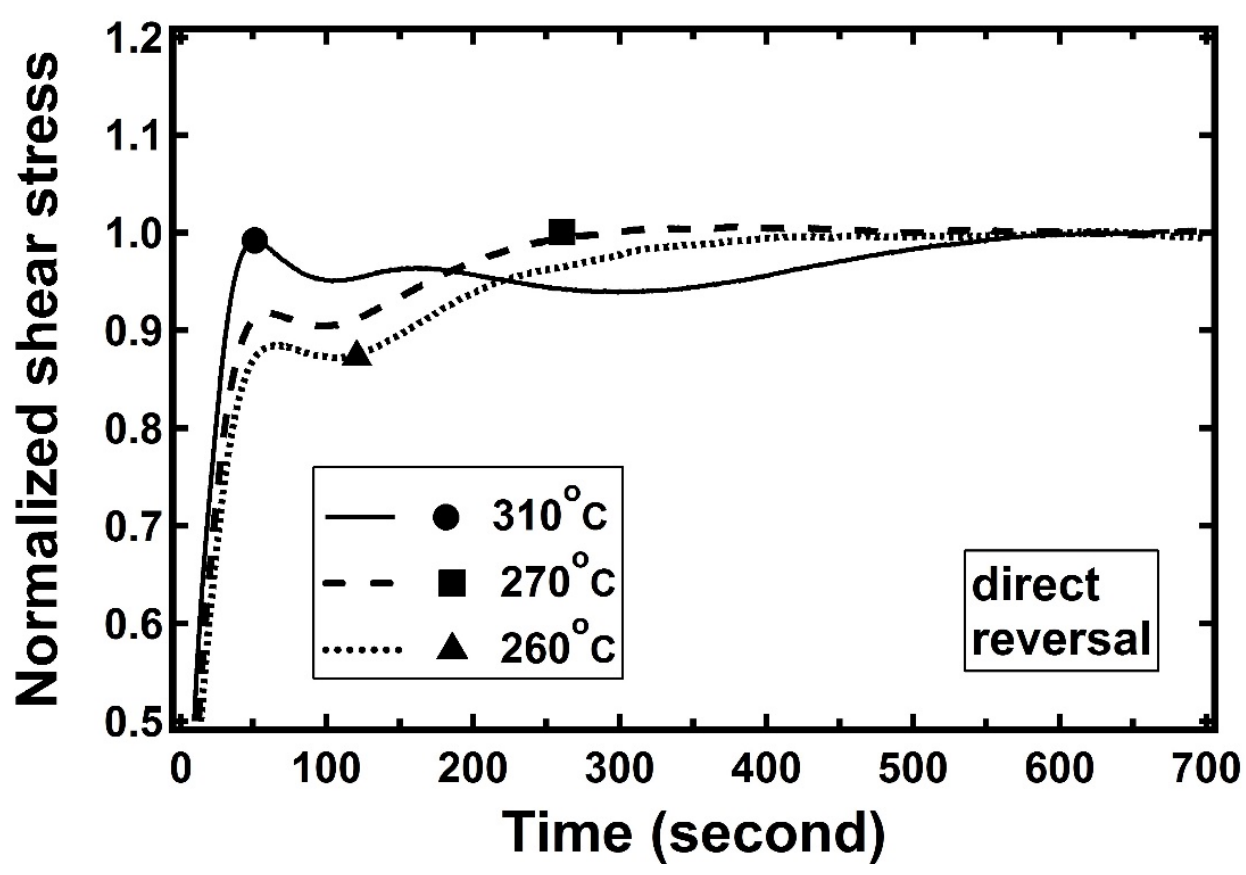

Figure 16: Trace of normalized transient shear stress in flow reversal. Reversed shear was directly applied without relaxation after the TLCP was sheared to steady state. Measurements were performed at 310,270 and $260^{\circ} \mathrm{C}$. 


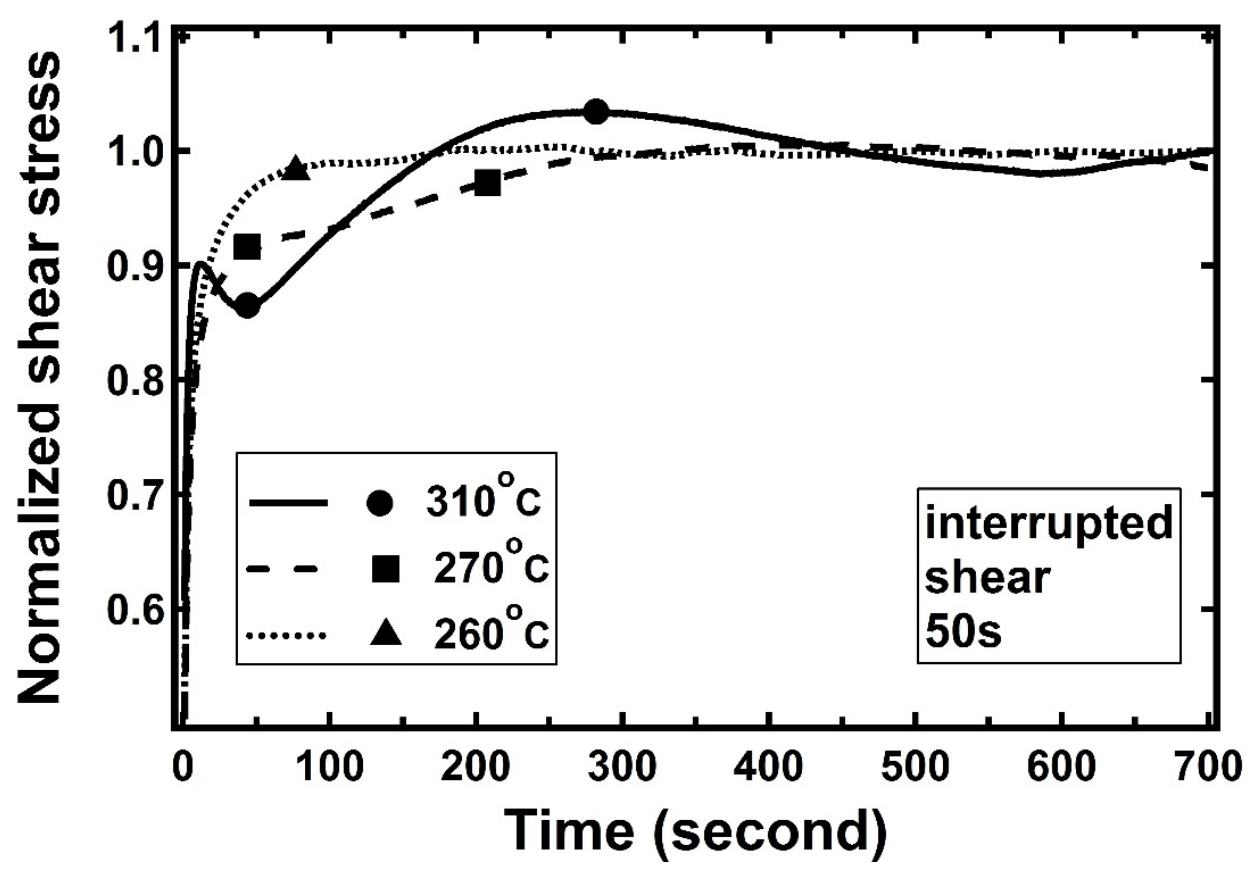

Figure 17: Trace of normalized transient shear stress in flow reversal after $50 \mathrm{~s}$ of relaxation. Measurements were performed at 310,270 and $260^{\circ} \mathrm{C}$.

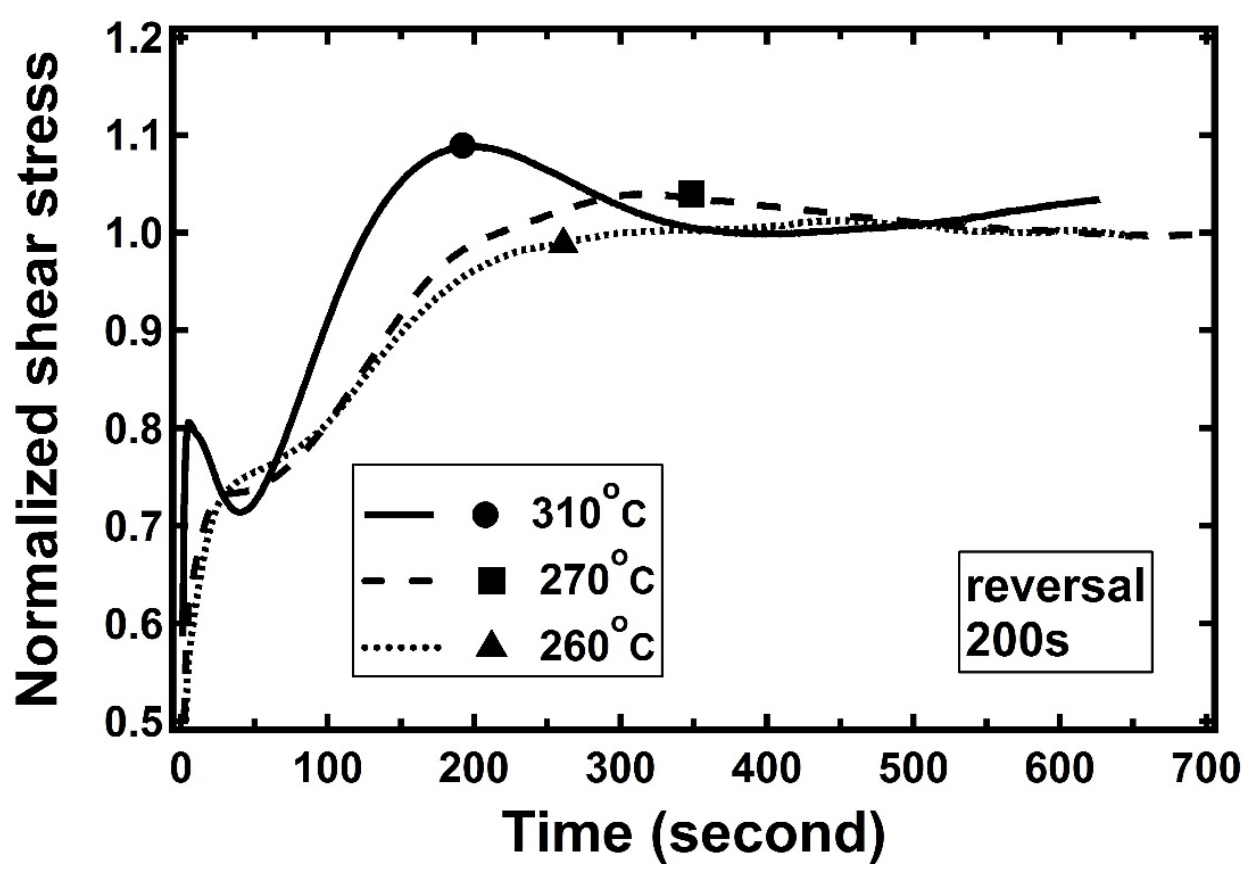


Figure 18: Trace of normalized transient shear stress in flow reversal after $200 \mathrm{~s}$ of relaxation. Measurements were performed at 310,270 and $260^{\circ} \mathrm{C}$.

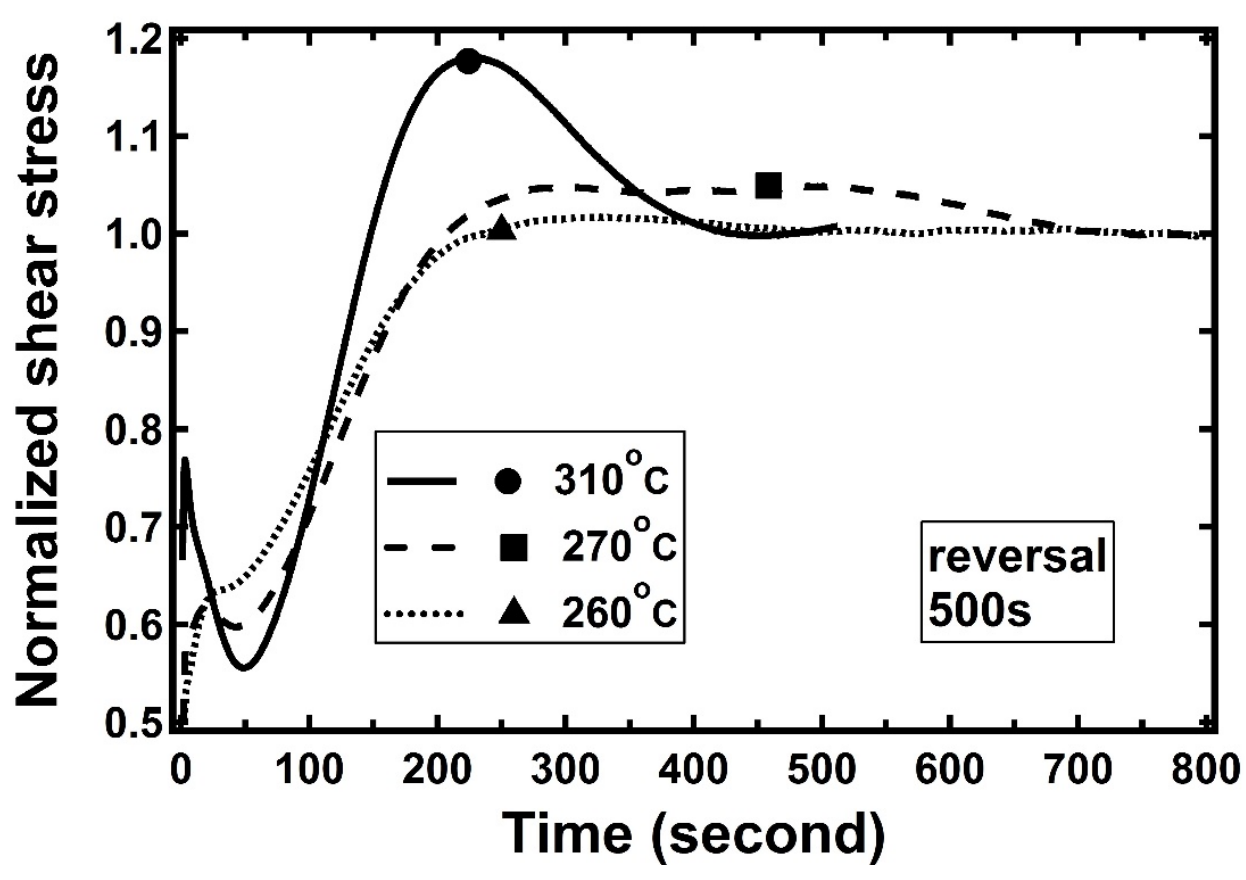

Figure 19: Trace of normalized transient shear stress in flow reversal after $500 \mathrm{~s}$ of relaxation. Measurements were performed at 310,270 and $260^{\circ} \mathrm{C}$.

\section{Conclusions}

The onset of crystallization of the liquid crystalline copolyester of HBA/TA/HQ/HQderivatives (HX8000) does not occur unless the polymer melt is cooled to at least $250^{\circ} \mathrm{C}$, which is $30^{\circ} \mathrm{C}$ below its melting point. This temperature serves as the lower temperature limit for the processing of the TLCP in the super-cooled state. The onset of crystallization is suggested by both small amplitude oscillatory shear tests and transient experiments at the startup of shear flow. Rheological measurements are more sensitive to detecting the onset of TLCP crystallization in the super-cooled state relative to DSC, and these measurements can be used on other TLCPs to guide 
processing in the super-cooled state. As the TLCP is cooled $30^{\circ} \mathrm{C}$ below its melting point, one order magnitude increase in viscosity is obtained at a shear rate of $0.1 \mathrm{~s}^{-1}$.

The transient rheology of HX8000 in the super-cooled state has been determined for the first time. Super cooling the liquid crystalline copolyester composed of HBA/TA/HQ/HQderivatives (HX8000) does not remarkably affect the relaxation of shear stress after preshearing. Nevertheless, in the interrupted shear measurements, the recovery of transient shear stress after preshearing is significantly suppressed in the super-cooled state.

\section{Acknowledgements}

Materials provided by Dupont are gratefully acknowledged. The authors acknowledge Prof. Dr. Garth L. Wilkes of Virginia Tech for the tremendous inputs and productive discussions. The authors would also like to thank the Department of Energy through the Savanah River National Laboratories for the financial support.

\section{References}

[1] H. G. Chae and S. Kumar, "Rigid-rod polymeric fibers", Journal of Applied Polymer Science, vol.100, pp 791-802, 2006.

[2] J. S. Chiou and D. R. Paul, "Gas transport in a thermotropic liquid-crystalline polyester", Journal of Polymer Science Part B: Polymer Physics, vol.25, pp 1699-1707, 1987.

[3] N. R. Miranda, J. T. Willits, B. D. Freeman and H. B. Hopfenberg, "Organic vapor sorption and transport in a thermotropic liquid crystalline polyester", Journal of Membrane Science, vol.94, pp 67-83, 1994.

[4] D. H. Weinkauf and D. R. Paul, "Gas transport properties of thermotropic liquid-crystalline copolyesters. II. The effects of copolymer composition", Journal of Polymer Science Part B: Polymer Physics, vol.30, pp 837-849, 1992.

[5] D. H. Weinkauf and D. R. Paul, "Gas transport properties of liquid crystalline poly (ethylene terephthalate-co-p-oxybenzoate)", Journal of Polymer Science Part B: Polymer Physics, vol.29, pp 329-340, 1991.

[6] D. H. Weinkauf and D. R. Paul, "Gas transport properties of thermotropic liquid-crystalline copolyesters. I. The effects of orientation and annealing", Journal of Polymer Science Part B: Polymer Physics, vol.30, pp 817-835, 1992.

[7] J. Lange and Y. Wyser, "Recent innovations in barrier technologies for plastic packaging - a review", Packaging Technology and Science, vol.16, pp 149-158, 2003. 
[8] Z. Li, Z. Zhou, S. R. Armstrong, E. Baer, D. R. Paul and C. J. Ellison, "Multilayer coextrusion of rheologically modified main chain liquid crystalline polymers and resulting orientational order", Polymer, vol.55, pp 4966-4975, 2014.

[9] K. Araki, T. Kitano, A. Lengalova and P. Saha, "Transient shear flow properties of carbon fiber-filled liquid crystalline polymer", Polymer Composites, vol.26, pp 470-476, 2005.

[10] T. K. Katsuhiko Araki, Berenika Hausnerova, "Rheological properties of carbon fiber and carbon black filled liquid crystalline polymer melts", Applied Rheology, vol.11, pp 188, 2001.

[11] J. A. King, T. M. Tambling, F. A. Morrison, J. M. Keith, A. J. Cole and R. M. Pagel, "Effects of carbon fillers on the rheology of highly filled liquid-crystal polymer based resins", Journal of Applied Polymer Science, vol.108, pp 1646-1656, 2008.

[12] Z. Li, P. A. Gonzalez Garza, E. Baer and C. J. Ellison, "Modification of rheological properties of a thermotropic liquid crystalline polymer by melt-state reactive processing", Polymer, vol.53, pp 3245-3252, 2012.

[13] C.F. Corallo, R.B. Sandor and R. S. Black, "Extrusion blow molding of thermotropic liquid crystalline polymers", U.S. Patent, 5336446,1992.

[14] C.F. Corallo, R.B. Sandor, R.S. Black and D. B. McKie, "Extrusion blow molding of filled liquid crystalline polymers", U.S. Patent, 5306461,1994.

[15] K. G. Blizard and D. G. Baird, "Blow molding of thermotropic liquid crystalline polyemrs", International Polymer Processing, vol.3, pp 172, 1989.

[16] K. G. Blizard, T. S. Wilson and D. G. Baird, "Film blowing of thermotropic liquidcrystalline polymers", International Polymer Processing, vol.5, pp 53-61, 1990.

[17] D. Done and D. G. Baird, "Solidification behavior and recovery kinetics of liquid crystlline polymers", Polymer Engineering and Science, vol.30, pp 989, 1990.

[18] F. Beekmans, A. D. Gotsis and B. Norder, "Influence of the flow history on stress growth and structure changes in the thermotropic liquid crystalline polymer Vectra B950", Rheologica Acta, vol.36, pp 82-95, 1997.

[19] F. Beekmans, A. D. Gotsis and B. Norder, "Transient and steady-state rheological behavior of the thermotropic liquid crystalline polymer Vectra B950", Journal of Rheology, vol.40, pp 947-966, 1996.

[20] T. De' Nève, P. Navard and M. Kléman, "Shear rheology and shear - induced textures of a thermotropic copolyesteramide", Journal of Rheology, vol.37, pp 515-529, 1993.

[21] T. Guo, G. M. Harrison and A. A. Ogale, "Transient shear rheology and rheo-optical microstructural characterization of a thermotropic liquid crystalline polymer", Polymer Engineering and Science, vol.45, pp 187-197, 2005.

[22] D. Done and D. G. Baird, "Transient flow of thermotropic liquid crystal polymers in step strain experiments", Journal of Rheology, vol.34, pp 749, 1990.

[23] H. C. Langelaan and A. D. Gotsis, "The relaxation of shear and normal stresses of nematic liquid crystalline polymers in squeezing and shear flows", Journal of Rheology, vol.40, pp 107-129, 1996.

[24] C. Dae Han and S. Chang, "Note: On the first normal stress difference of the thermotropic copolyester 73/27 HBA/HNA", Journal of Rheology, vol.38, pp 241-244, 1994.

[25] F. Cocchini, M. R. Nobile and D. Acierno, "Letter: About negative first normal stress differences in a thermotropic liquid crystalline polymer", Journal of Rheology, vol.36, pp 1307-1311, 1992. 
[26] F. Cocchini, M. R. Nobile and D. Acierno, "Transient and steady rheological behavior of the thermotropic liquid crystal copolymer 73/27 HBA/HNA", Journal of Rheology, vol.35, pp 1171-1189, 1991.

[27] G. G. Viola and D. G. Baird, "Studies on the transient shear flow behavior of liquid crystalline polymers", Journal of Rheology, vol.30, pp 601-628, 1986.

[28] S. M. Guskey and H. H. Winter, "Transient shear behavior of a thermotropic liquidcrystalline polymer in the nematic state", Journal of Rheology, vol.35, pp 1191-1207, 1991.

[29] S. Rendon, W. Burghardt and R. Bubeck, "Orientation dynamics in commercial thermotropic liquid crystalline polymers in transient shear flows", Rheologica Acta, vol.46, pp 945-956, 2007.

[30] A. D. Gotsis and D. G. Baird, "Primary normal-stress difference for two liquid-crystalline copolyesters", Rheologica Acta, vol.25, pp 275-286, 1986.

[31] A. Donald, A. Windle and S. Hanna, Liquid Crystalline Polymers, Cambridge University, 2006.

[32] M. A. McLeod and D. G. Baird, "The crystallization behavior of blends of thermotropic liquid crystalline polymers", Polymer, vol.40, pp 3743-3752, 1999.

[33] C. M. McCullagh, J. Blackwell and A. M. Jamieson, "X-ray Analysis of the Kinetics of Transesterification in Blends of Wholly Aromatic Thermotropic Copolyesters", Macromolecules, vol.30, pp 4837-4844, 1997.

[34] C. M. McCullagh, J. Blackwell and A. M. Jamieson, "Transesterification in Blends of Wholly Aromatic Thermotropic Copolyesters", Macromolecules, vol.27, pp 2996-3001, 1994.

[35] D. G. Baird and D. I. Collias, Polymer Processing Principles and Design, Section 5.4, Second edition, 2013.

[36] C. Qian, "Connecting the rheological behavior to the processing of thermotropic liquid crystalline polymers in the super-cooled state", PhD dissertation, Virginia Tech, 2016.

[37] Y. G. Lin and H. H. Winter, "High-temperature recrystallization and rheology of a thermotropic liquid crystalline polymer", Macromolecules, vol.24, pp 2877-2882, 1991.

[38] S. G. Baek, J. J. Magda, R. G. Larson and S. D. Hudson, "Rheological differences among liquid - crystalline polymers. II. Disappearance of negative N1 in densely packed lyotropes and thermotropes", Journal of Rheology, vol.38, pp 1473-1503, 1994.

[39] S. G. Baek, J. J. Magda and R. G. Larson, "Rheological differences among liquidcrystalline polymers. I. The first and second normal stress differences of PBG solutions", Journal of Rheology, vol.37, pp 1201-1224, 1993.

[40] G. Marrucci and P. L. Maffettone, "A description of the liquid-crystalline phase of rodlike polymers at high shear rates", Macromolecules, vol.22, pp 4076-4082, 1989.

[41] D. S. Kalika, D. W. Giles and M. M. Denn, "Shear and time - dependent rheology of a fully nematic thermotropic liquid crystalline copolymer", Journal of Rheology, vol.34, pp 139-154, 1990.

[42] W. R. Burghardt and G. G. Fuller, "Role of director tumbling in the rheology of polymer liquid crystal solutions", Macromolecules, vol.24, pp 2546-2555, 1991. 